\title{
ASYMPTOTIC RISK COMPARISON OF IMPROVED ESTIMATORS FOR NORMAL COVARIANCE MATRIX
}

\author{
By
}

\author{
Nariaki Sugiura and Masahiro Fujimoto
}

Asymptotic risks of the empirical Bayes estimators $\hat{\Sigma}_{H}$ by Haff [5] for a covariance matrix $\Sigma$ in a $p$-dimensional normal distribution are computed and compared with that of James and Stein's minimax estimators $\hat{\Sigma}_{J S}$. For $p \geqq 6$, it is shown that $\hat{\Sigma}_{J S}$ are always better than $\hat{\Sigma}_{H}$ asymptotically, though the leading terms are the same. New estimators which dominate $\hat{\Sigma}_{J S}$ for some $\Sigma$ in any $p$ asymptotically are proposed. Some numerical comparisons are given. Exact risks for ordinary estimators $\hat{\Sigma}_{O}$ and minimax estimators $\hat{\Sigma}_{J S}$ are also computed and compared with asymptotic ones for which the approximations are shown to be excellent.

\section{Introduction}

Let $S$ have a Wishart distribution with unknown scale matrix $\Sigma$ and $n$ degrees of freedom, for which we shall write $S: W_{p}(n, \Sigma)$ and assume $n>p+1$. Let $\hat{\Sigma}$ be an estimator of $\Sigma$. The loss function is taken to be

$$
L_{1}(\hat{\Sigma}, \Sigma)=\operatorname{tr} \hat{\Sigma} \Sigma^{-1}-\log \left|\hat{\Sigma} \Sigma^{-1}\right|-p
$$

or

$$
L_{2}(\hat{\Sigma}, \Sigma)=\frac{1}{2} \operatorname{tr}\left(\hat{\Sigma} \Sigma^{-1}-I\right)^{2} .
$$

The $L_{1}$ loss is equivalent to the likelihood ratio statistic for testing the hypothesis $\Sigma=\Sigma_{0}$ against all alternatives. The $L_{2}$ loss can also be used as a test statistic for the same problem as in Nagao [10]. The factor $1 / 2$ in the $L_{2}$ loss is not essential. However we wish to retain it, since $L_{1}$ loss tends to $\operatorname{tr}\left(\hat{\Sigma} \Sigma^{-1}-I\right)^{2} / 2$, when $\hat{\Sigma}$ is close to $\Sigma$. The risk function is given by $R_{i}(\hat{\Sigma}, \Sigma)=E\left[L_{i}(\hat{\Sigma}, \Sigma)\right]$ for $i=1$ or 2 . Haff [5] proved that among the scalar multiples of $S$, the best estimator under $L_{1}$ is $\hat{\Sigma}_{o}^{(1)}=S / n$ and that under $L_{2}$ it is given by $\hat{\Sigma}_{o}^{(2)}=S /(n+p+1)$, which we call ordinary estimators. Then he considered the posterior mean of $\Sigma$ for a prior distribution $W_{p}\left[n^{\prime},(\gamma C)^{-1}\right]$ for $\Sigma^{-1}$ with unknown scalar $\gamma>0$ and known p.d. matrix

Received October 13, 1981. Revised February 15, 1982. 
$C$. It is given by $E[\Sigma \mid S, \gamma]=(S+\gamma C) /\left(n+n^{\prime}-p-1\right)$. In the process of estimating $\gamma$ by maximizing approximate marginal likelihood of $S$, he obtained $u t(u)$ for $u=$ $1 / \operatorname{tr}\left(S^{-1} C\right)$ as an estimator for $\gamma$, where $t(\cdot)$ is nonincreasing. He then proved that under $L_{1}$ the estimator

$$
\hat{\Sigma}_{H}^{(1)}=\frac{1}{n}[S+u t(u) C]
$$

for $0 \leqq t(u) \leqq 2(p-1) / n$, dominates $\hat{\Sigma}_{o}^{(1)}=S / n$ for any $n>p+1$ and under $L_{2}$ the estimator

$$
\hat{\Sigma}_{H}^{(2)}=\frac{1}{n+p+1}(S+u t C)
$$

for $0 \leqq t \leqq 2(p-1) /(n-p+3)$, dominates $\hat{\Sigma}_{o}^{(2)}=S /(n+p+1)$ for any $n>p+1$. It was also shown that if $t(u)$ in $(1.3)$ is constant, the best choice of $t(u)$ is $(p-1) / n$ and that the best choice of $t$ in $(1.4)$ is $(p-1) /(n-p+3)$. In this paper we always take these optimal values for $t$ and call them Haff's estimators $\hat{\Sigma}_{H}^{(1)}$ and $\hat{\Sigma}_{H}^{(2)}$ respectively.

A minimax estimator for $\Sigma$ was earlier obtained by James and Stein [7], giving

$$
\hat{\Sigma_{J S}^{(i)}}=K \Delta^{(i)} K^{\prime}
$$

for the loss $L_{i}$ ( $i=1$ or 2 ), where the lower triangular matrix $K$ with positive diagonal elements is obtained from $S=K K^{\prime}$ and $\Delta^{(i)}=\operatorname{diag}\left[\Delta_{1}^{(i)}, \cdots, \Delta_{p}^{(i)}\right]$. For the $L_{1}$ loss, they proved that $\Delta_{j}^{(1)}=1 /(n+p+1-2 j)$ and reported that they were unable to get explicit form of $\Delta_{j}^{(2)}$. Sharma [13] derived the linear equations for $\Delta_{j}^{(2)}$, from which numerical values are computed for given $n$ and $p$. They were also obtained earlier by Selliah [12],

The primary purpose of this paper is to compare the asymptotic risk of Haff's estimator $\hat{\Sigma}_{H}^{(i)}$ with that of James and Stein's estimator $\hat{\Sigma}_{J S}^{(i)}$ under $L_{i}$ for $i=1$ or 2 . Under $L_{2}$, we have derived an asymptotic form of $\Delta_{j}^{(2)}$ for large $n$. It is shown that the leading terms of the asymptotic risks for $\hat{\Sigma}_{H}^{(i)}$ and $\hat{\Sigma}_{J S}^{(i)}$ are the same and that the next term for $\hat{\Sigma}_{H}^{(i)}$ is less than that of $\hat{\Sigma}_{J S}^{(i)}$ only for $2 \leqq p \leqq 5$ and for some $\Sigma$. If $p \geqq 6$, the second term of the asymptotic expansion of $R_{i}\left(\hat{\Sigma}_{H}^{(i)}, \Sigma\right)$ is always larger than that of $R_{i}\left(\hat{\Sigma}_{J S}^{(i)}, \Sigma\right)$ for all $\Sigma$.

Secondly we shall propose new estimators for $\Sigma$ by minimizing risks empirically, which are given by

$$
\hat{\Sigma}^{(1)}=\frac{1}{n}\left[S+b \frac{\operatorname{tr} C S^{-1}}{\operatorname{tr}\left(C S^{-1}\right)^{2}} C\right], \quad 0 \leqq b \leqq \frac{2(p-1)}{n}
$$

for $L_{1}$ loss and

$$
\hat{\Sigma}^{(2)}=\frac{1}{n+p+1}\left[S+b \frac{\operatorname{tr} C S^{-1}}{\operatorname{tr}\left(C S^{-1}\right)^{2}} C\right], \quad 0 \leqq b \leqq \frac{2(p-1)}{n}
$$


for $L_{2}$ loss. It is shown that our new estimator $\hat{\Sigma}^{(1)}$ dominates $\hat{\Sigma}_{o}^{(1)}$ for all $n>p+1$ and that $\hat{\Sigma}^{(2)}$ dominates $\hat{\Sigma}_{0}^{(2)}$ asymptotically. The result also holds for more general form of $\hat{\Sigma}^{(1)}$, that is, the constant $b$ in (1.6) can be replaced by $t(\cdot)$ in (1.3) for $u=\operatorname{tr} C S^{-1} / \operatorname{tr}\left(C S^{-1}\right)^{2}$. However we prefer to (1.6) to simplify later discussions. The leading term of the asymptotic risk is the same as that of $\hat{\Sigma}_{J S}^{(i)}$ and the second term is less than that of $\hat{\Sigma}_{J S}^{(i)}$ for some $\Sigma$ and for all $p>1$. Eliminating the leading term, the range of $R_{i}\left(\hat{\Sigma}^{(i)}, \Sigma\right)$ is much wider below than $R_{i}\left(\hat{\Sigma}_{H}^{(i)}, \Sigma\right)$ asymptotically. However the absolute difference $R_{i}\left(\hat{\Sigma}^{(i)}, \Sigma\right)-R_{i}\left(\hat{\Sigma}_{J S}^{(i)}, \Sigma\right)$ or $R_{i}\left(\hat{\Sigma}_{H}^{(i)}, \Sigma\right)-R_{i}\left(\hat{\Sigma}_{J S}^{(i)}, \Sigma\right)$ is not so large.

To get some idea for the errors of asymptotic approximations, the terms of order $n^{-3}$ (third terms) are computed for $R_{i}\left(\hat{\Sigma}_{H}^{(i)}, \Sigma\right)$ and $R_{i}\left(\hat{\Sigma}^{(i)}, \Sigma\right)$. The exact risks of $\hat{\Sigma}_{J S}^{(i)}$ are computed and asymptotic values up to order $n^{-3}$ are compared. For $2 \leqq p \leqq 6$ and $n \geqq 16$, asymptotic values for $\hat{\Sigma}_{J S}^{(i)}$ are accurate for three (two) significant digits for $L_{1}\left(L_{2}\right)$ loss in most cases examined. The rates of the reduction of the risks of $\hat{\Sigma}_{H}^{(i)}\left(\hat{\Sigma}^{(i)}\right)$ with respect to $\hat{\Sigma}_{o}^{(i)}$ are shown to be the highest $8 \%(20 \%)$ for $i=1, n \geqq 16$ and $4 \%(11 \%)$ for $i=2, n \geqq 32$ respectively within our examples computed in Tables.

\section{Derivation of new estimators}

Since our goal is to find an estimator $\hat{\Sigma}$ which minimizes the risk, we shall look for a solution in a form $\hat{\Sigma}^{(1)}=(S+\gamma C) / n$ for $L_{1}$ or $\hat{\Sigma}^{(2)}=(S+\gamma C) /(n+p+1)$ for $L_{2}$. The risk for $L_{1}$ is given by

$$
R_{1}\left(\hat{\Sigma}^{(1)}, \Sigma\right)=\frac{\gamma}{n} \operatorname{tr} C \Sigma^{-1}-E\left[\log \left|\frac{1}{n}(S+\gamma C) \Sigma^{-1}\right|\right] .
$$

Hence the derivative with respect to $\gamma$ is

$$
\frac{1}{n} \operatorname{tr} C \Sigma^{-1}-E\left[\operatorname{tr}\left(\gamma I+S C^{-1}\right)^{-1}\right]
$$

where the expectation is taken by $S$ having $W_{p}(n, \Sigma)$ distribution. At $\gamma=0$, the derivative has a negative value $-(p+1) \operatorname{tr} C \Sigma^{-1} /\{n(n-p-1)\}$, since $E\left(S^{-1}\right)=\Sigma^{-1} /(n-$ $p-1)$, by Kshirsagar [9], for example. This shows that the risk will be smaller if we take $\gamma$ positive near zero. Assume that $\gamma$ is small and put the derivative (2.2) equal to zero. We get an equation for $\gamma$, an approximate solution of which is given by

$$
\gamma=(p+1) \frac{\operatorname{tr} C \Sigma^{-1}}{\operatorname{tr}\left(C \Sigma^{-1}\right)^{2}}
$$

which yields the estimator (1.6). The estimator (1.7) for $L_{2}$ is similarly derived. 
The constant factor $b$ is restricted so that it dominates ordinary estimator $\hat{\Sigma}_{o}^{(i)}$, which will be discussed later.

\section{Risks of ordinary and James and Stein's minimax estimators}

Using the Bartlett's decomposition (Giri [3], page 126) of Wishart matrix $S$ when $\Sigma=I$, we get

$$
R_{1}\left(\hat{\Sigma}_{O}^{(1)}, \Sigma\right)=p \log n-\sum_{j=1}^{p} E\left[\log \chi_{n-j+1}^{2}\right]
$$

where $\chi_{m}^{2}$ denotes the $\chi^{2}$ variate with $m$ degrees of freedom. Using digamma function $\psi(x)=d \log \Gamma(x) / d x$, we can rewrite it

$$
p \log \frac{n}{2}-\sum_{j=1}^{p} \phi\left(\frac{n-j+1}{2}\right)
$$

If $n$ is an integer larger than one, we know that

$$
\psi(n)=1+\frac{1}{2}+\cdots+\frac{1}{n-1}-\gamma
$$

for Euler's constant $\gamma=0.5772156649015329 \cdots$ (Abramowitz and Stegun[[1]). For half integer argument $(n \geqq 1)$,

$$
\phi\left(n+\frac{1}{2}\right)=-r-2 \log 2+2\left(1+\frac{1}{3}+\cdots+\frac{1}{2 n-1}\right) .
$$

These are sufficient for the computation of $R_{1}\left(\hat{\Sigma}_{o}^{(1)}, \Sigma\right)$. If $n$ is large, an asymptotic formula for $\phi$ is available, which is derived from Stirling's formula (Kendall [8], page 245)

$$
\psi(x+h)=\log x+\frac{h-1 / 2}{x}+\sum_{r=1}^{n} \frac{(-1)^{r} B_{r+1}(h)}{x^{r+1}(r+1)}+O\left(\frac{1}{x^{n+2}}\right),
$$

where $B_{r}(h)$ are the Bernoulli polynomials given by $B_{2}(h)=h^{2}-h+1 / 6, B_{3}(h)=h^{3}-$ $(3 / 2) h^{2}+(1 / 2) h$. This yields

$$
R_{1}\left(\hat{\Sigma}_{o}^{(1)}, \Sigma\right)=\frac{p(p+1)}{2 n}+\frac{p\left(2 p^{2}+3 p-1\right)}{12 n^{2}}+\frac{p\left(p^{2}-1\right)(p+2)}{12 n^{3}}+O\left(n^{-4}\right) .
$$

Some numerical values of $R_{1}\left(\hat{\Sigma}_{0}^{(1)}, \Sigma\right)$ are computed based on $(3.2) \sim(3.4)$ and compared with the asymptotic values (3.6) for $p=2 \sim 6$ and $n=8 \sim 128$. They are shown in Table 1 . We can see that the asymptotic approximations are excellent, namely, for $n \geqq 16$ and $p \leqq 6$, the values are accurate with three significance digits.

Under $L_{2}$ loss, Haff [5] noted that

$$
R_{2}\left(\hat{\Sigma}_{o}^{(2)}, \Sigma\right)=\frac{p(p+1)}{2(n+p+1)},
$$


Table 1. Values of $R_{1}\left(\hat{\Sigma}_{0}^{(1)}, \Sigma\right)$

\begin{tabular}{|c|c|c|c|c|c|c|}
\hline & & $n=8$ & $n=16$ & $n=32$ & $n=64$ & $n=128$ \\
\hline$p=2$ & $\begin{array}{l}O\left(n^{-1}\right) \\
O\left(n^{-2}\right) \\
O\left(n^{-3}\right)\end{array}$ & $\begin{array}{l}.37500 \\
.03385 \\
.00391\end{array}$ & $\begin{array}{l}.187500 \\
.008464 \\
.000488\end{array}$ & $\begin{array}{l}.093750 \\
.002116 \\
.000061\end{array}$ & $\begin{array}{l}.046875 \\
.000529 \\
.0000008\end{array}$ & $\begin{array}{l}.023438 \\
.000132 \\
.000001\end{array}$ \\
\hline & $\begin{array}{r}\text { approx. } \\
\text { exact }\end{array}$ & $\begin{array}{l}.4128 \\
.413314\end{array}$ & $\begin{array}{l}.19645 \\
.196484\end{array}$ & $\begin{array}{l}.095927 \\
.095929\end{array}$ & $\begin{array}{l}.047412 \\
.047412\end{array}$ & $\begin{array}{l}.023571 \\
.023571\end{array}$ \\
\hline$p=3$ & $\begin{array}{l}O\left(n^{-1}\right) \\
O\left(n^{-2}\right) \\
O\left(n^{-3}\right)\end{array}$ & $\begin{array}{l}.75000 \\
.10156 \\
.01953\end{array}$ & $\begin{array}{l}.37500 \\
.02539 \\
.00244\end{array}$ & $\begin{array}{l}.187500 \\
.006348 \\
.000305\end{array}$ & $\begin{array}{l}.093750 \\
.001587 \\
.000038\end{array}$ & $\begin{array}{l}.046875 \\
.000397 \\
.000005\end{array}$ \\
\hline & $\begin{array}{r}\text { approx. } \\
\text { exact }\end{array}$ & $\begin{array}{l}.871 \\
.876824\end{array}$ & $\begin{array}{l}.4028 \\
.403141\end{array}$ & $\begin{array}{l}.19415 \\
.194171\end{array}$ & $\begin{array}{l}.095375 \\
.095376\end{array}$ & $\begin{array}{l}.047276 \\
.047277\end{array}$ \\
\hline$p=4$ & $\begin{array}{l}O\left(n^{-1}\right) \\
O\left(n^{-2}\right) \\
O\left(n^{-3}\right)\end{array}$ & $\begin{array}{r}1.2500 \\
.2240 \\
.0586\end{array}$ & $\begin{array}{l}.62500 \\
.05599 \\
.00732\end{array}$ & $\begin{array}{l}.312500 \\
.013997 \\
.000916\end{array}$ & $\begin{array}{l}.156250 \\
.003499 \\
.000114\end{array}$ & $\begin{array}{l}.078125 \\
.000875 \\
.000014\end{array}$ \\
\hline & $\begin{array}{r}\text { approx. } \\
\text { exact }\end{array}$ & $\begin{array}{l}1.533 \\
1.559962\end{array}$ & $\begin{array}{l}.6883 \\
.689672\end{array}$ & $\begin{array}{l}.32741 \\
.327490\end{array}$ & $\begin{array}{l}.159864 \\
.159868\end{array}$ & $\begin{array}{l}.079014 \\
.079015\end{array}$ \\
\hline$p=5$ & $\begin{array}{l}O\left(n^{-1}\right) \\
O\left(n^{-2}\right) \\
O\left(n^{-3}\right)\end{array}$ & $\begin{array}{r}1.8750 \\
.4167 \\
.1367\end{array}$ & $\begin{array}{l}.9375 \\
.1042 \\
.0171\end{array}$ & $\begin{array}{l}.46875 \\
.02604 \\
.00214\end{array}$ & $\begin{array}{l}.234375 \\
.006510 \\
.000267\end{array}$ & $\begin{array}{l}.117188 \\
.001628 \\
.000033\end{array}$ \\
\hline & $\begin{array}{r}\text { approx. } \\
\text { exact }\end{array}$ & $\begin{array}{l}2.43 \\
2.52347\end{array}$ & $\begin{array}{l}1.059 \\
1.06300\end{array}$ & $\begin{array}{l}.4969 \\
.497161\end{array}$ & $\begin{array}{l}.24115 \\
.241166\end{array}$ & $\begin{array}{l}.118848 \\
.118849\end{array}$ \\
\hline$p=6$ & $\begin{array}{l}O\left(n^{-1}\right) \\
O\left(n^{-2}\right) \\
O\left(n^{-3}\right)\end{array}$ & $\begin{array}{r}2.6250 \\
.6953 \\
.2734\end{array}$ & $\begin{array}{r}1.3125 \\
.1738 \\
.0342\end{array}$ & $\begin{array}{l}.65626 \\
.04346 \\
.00427\end{array}$ & $\begin{array}{l}.328125 \\
.010864 \\
.000534\end{array}$ & $\begin{array}{l}.164063 \\
.002716 \\
.000067\end{array}$ \\
\hline & $\underset{\text { exact }}{\text { approx. }}$ & $\begin{array}{l}3.59 \\
3.87328\end{array}$ & $\begin{array}{l}1.521 \\
1.53134\end{array}$ & $\begin{array}{l}.7040 \\
.704554\end{array}$ & $\begin{array}{l}.33952 \\
.339557\end{array}$ & $\begin{array}{l}.166845 \\
.166847\end{array}$ \\
\hline
\end{tabular}

which is asymptotically the same as $R_{1}\left(\hat{\Sigma}_{O}^{(1)}, \Sigma\right)$ for large $n$. This is the reason why we prefer multiplier $1 / 2$ in the definition of $L_{2}$ loss in (1.2). Unlike the simple form of (3.7), the asymptotic approximations

$$
R_{2}\left(\hat{\Sigma}_{o}^{(2)}, \Sigma\right)=\frac{p(p+1)}{2 n}-\frac{p(p+1)^{2}}{2 n^{2}}+\frac{p(p+1)^{3}}{2 n^{3}}+O\left(n^{-4}\right)
$$

are not so excellent as $R_{1}\left(\hat{\Sigma}_{o}^{(1)}, \Sigma\right)$. For example, the exact value of $R_{2}\left(\hat{\Sigma}_{o}^{(2)}, \Sigma^{\prime}\right)$ in (3.7) for $p=2$ and $n=16$ is 0.15789 , while the asymptotic value of (3.8) gives 0.15894 which is accurate for three significant digits. From Table 1, the corresponding exact value of $R_{1}\left(\hat{\Sigma}_{o}^{(1)}, \Sigma\right)$ is 0.19648 and the asymptotic value is 0.19645 which is accurate for one more digit than $R_{2}\left(\hat{\Sigma}_{O}^{(2)}, \Sigma\right)$. This is the case with other values of parameters $n$ and $p$.

Next we shall evaluate the risks of the minimax estimators by James and Stein [7], By considering a best equivariant estimator $\phi\left(L S L^{\prime}\right)=L \phi(S) L^{\prime}$ for the transformation group of lower triangular matrices $L$ with positive diagonal elements, they obtained a minimax estimator of (1.5) under $L_{1}$ loss and derived

$$
R_{1}\left(\hat{\Sigma}_{J S}^{(1)}, \Sigma\right)=\sum_{j=1}^{p} \log (n+p-2 j+1)-\sum_{j=1}^{p} E\left[\log \chi_{n-j+1}^{2}\right]
$$


Using digamma function $\psi(x)$, this can be simplified as

$$
\sum_{j=1}^{p} \log \frac{1}{2}(n+p-2 j+1)-\sum_{j=1}^{p} \phi\left(\frac{n-j+1}{2}\right),
$$

which is useful for numerical computations. The asymptotic form of (3.10) is obtained by (3.5), giving

$$
R_{1}\left(\hat{\Sigma}_{J S}^{(1)}, \nu^{\prime}\right)=\frac{p(p+1)}{2 n}+\frac{p(3 p+1)}{12 n^{2}}+\frac{p\left(p^{2}-1\right)(p+2)}{12 n^{3}}+O\left(n^{-4}\right) .
$$

In Table 2 exact and asymptotic values of $R_{1}\left(\hat{\Sigma}_{J S}^{(1)}, \nu\right)$ are compared. It is found that for $n \geqq 16$ and $p \leqq 6$, the asymptotic values are accurate for three significant digits, which is the same conclusion as for $R_{1}\left(\hat{\Sigma}_{O}^{(1)}, \Sigma\right)$. Since equivariant estimators contain best scalar multiple of $S$, namely, $\hat{\Sigma}_{o}^{(1)}$, inequality $R_{1}\left(\hat{\Sigma}_{J S}^{(1)}, \Sigma^{\prime}\right)<R_{1}\left(\hat{\Sigma}_{o}^{(1)}, \Sigma^{\prime}\right)$ holds as a matter of fact. If we take difference of the risks by asymptotic form, we get

$$
R_{1}\left(\hat{\Sigma}_{J S}^{(1)}, \nu^{\prime}\right)-R_{1}\left(\hat{\Sigma}_{O}^{(1)}, \nu^{\prime}\right)=-\frac{p\left(p^{2}-1\right)}{6 n^{2}}+O\left(n^{-4}\right),
$$

\begin{tabular}{|c|c|c|c|c|c|c|}
\hline & & $n=8$ & $n=16$ & $n=32$ & $n=64$ & $n=128$ \\
\hline$p=2$ & $\begin{array}{l}O\left(n^{-1}\right) \\
O\left(n^{-2}\right) \\
O\left(n^{-3}\right)\end{array}$ & $\begin{array}{l}.37500 \\
.01823 \\
.00391\end{array}$ & $\begin{array}{l}.187500 \\
.004557 \\
.000488\end{array}$ & $\begin{array}{l}.093750 \\
.001139 \\
.000061\end{array}$ & $\begin{array}{l}.046875 \\
.000285 \\
.000008\end{array}$ & $\begin{array}{l}.023438 \\
.000071 \\
.000001\end{array}$ \\
\hline & $\begin{array}{c}\text { approx. } \\
\text { exact }\end{array}$ & $\begin{array}{l}.3971 \\
.39757\end{array}$ & $\begin{array}{l}.19255 \\
.19257\end{array}$ & $\begin{array}{l}.094950 \\
.094952\end{array}$ & $\begin{array}{l}.047167 \\
.047168\end{array}$ & $\begin{array}{l}.023510 \\
.023510\end{array}$ \\
\hline$p=3$ & $\begin{array}{l}O\left(n^{-1}\right) \\
O\left(n^{-2}\right) \\
O\left(n^{-3}\right)\end{array}$ & $\begin{array}{l}.75000 \\
.03906 \\
.01953\end{array}$ & $\begin{array}{l}.37500 \\
.00977 \\
.00244\end{array}$ & $\begin{array}{l}.187500 \\
.002441 \\
.000305\end{array}$ & $\begin{array}{l}.093750 \\
.000610 \\
.000038\end{array}$ & $\begin{array}{l}.046875 \\
.000153 \\
.000005\end{array}$ \\
\hline & $\begin{array}{l}\text { approx. } \\
\text { exact }\end{array}$ & $\begin{array}{l}.809 \\
.81229\end{array}$ & $\begin{array}{l}.3872 \\
.38739\end{array}$ & $\begin{array}{l}.19025 \\
.190257\end{array}$ & $\begin{array}{l}.094398 \\
.094399\end{array}$ & $\begin{array}{l}.047033 \\
.047032\end{array}$ \\
\hline$p=4$ & $\begin{array}{l}O\left(n^{-1}\right) \\
O\left(n^{-2}\right) \\
O\left(n^{-3}\right)\end{array}$ & $\begin{array}{r}1.2500 \\
.0677 \\
.0586\end{array}$ & $\begin{array}{l}.62500 \\
.01693 \\
.00732\end{array}$ & $\begin{array}{l}.312500 \\
.004232 \\
.000916\end{array}$ & $\begin{array}{l}.156250 \\
.001058 \\
.000114\end{array}$ & $\begin{array}{l}.078125 \\
.000265 \\
.000014\end{array}$ \\
\hline & $\begin{array}{l}\text { approx. } \\
\text { exact }\end{array}$ & $\begin{array}{l}1.376 \\
1.3927\end{array}$ & $\begin{array}{l}.6493 \\
.64997\end{array}$ & $\begin{array}{l}.31765 \\
.31768\end{array}$ & $\begin{array}{l}.157422 \\
.157425\end{array}$ & $\begin{array}{l}.078404 \\
.078404\end{array}$ \\
\hline$p=5$ & $\begin{array}{l}O\left(n^{-1}\right) \\
O\left(n^{-2}\right) \\
O\left(n^{-3}\right)\end{array}$ & $\begin{array}{r}1.8750 \\
.1042 \\
.1367\end{array}$ & $\begin{array}{l}.9375 \\
.0260 \\
.0171\end{array}$ & $\begin{array}{l}.46875 \\
.00651 \\
.00214\end{array}$ & $\begin{array}{l}.234375 \\
.001628 \\
.000267\end{array}$ & $\begin{array}{l}.117188 \\
.000407 \\
.000033\end{array}$ \\
\hline & $\begin{array}{l}\text { approx. } \\
\text { exact }\end{array}$ & $\begin{array}{l}2.12 \\
2.1713\end{array}$ & $\begin{array}{l}.981 \\
.98271\end{array}$ & $\begin{array}{l}.4774 \\
.47750\end{array}$ & $\begin{array}{l}.236270 \\
.236275\end{array}$ & $\begin{array}{l}.117628 \\
.117628\end{array}$ \\
\hline$p=6$ & $\begin{array}{l}O\left(n^{-1}\right) \\
O\left(n^{-2}\right) \\
O\left(n^{-3}\right)\end{array}$ & $\begin{array}{r}2.6250 \\
.1484 \\
.2734\end{array}$ & $\begin{array}{r}1.3125 \\
.0371 \\
.0342\end{array}$ & $\begin{array}{l}.65625 \\
.00928 \\
.00427\end{array}$ & $\begin{array}{l}.328125 \\
.002319 \\
.000534\end{array}$ & $\begin{array}{l}.164063 \\
.000580 \\
.000067\end{array}$ \\
\hline & $\begin{array}{r}\text { approx. } \\
\text { exact }\end{array}$ & $\begin{array}{l}3.05 \\
3.2107\end{array}$ & $\begin{array}{l}1.384 \\
1.3889\end{array}$ & $\begin{array}{l}.6698 \\
.67003\end{array}$ & $\begin{array}{l}.33098 \\
.330991\end{array}$ & $\begin{array}{l}.164709 \\
.164710\end{array}$ \\
\hline
\end{tabular}

which is negative for $p \geqq 2$, neglecting the higher order terms. This suggests the

Table 2. Exact and asymptotic values of $R_{1}\left(\hat{\Sigma}_{J}^{(1)}, \Sigma^{\prime}\right)$ 
validity of the asymptotic comparisons.

Under $L_{2}$ loss, the exact $\Delta^{(2)}$ is not available. However Selliah [12] and Sharma [13] show that $\Delta=\left[\Delta_{1}^{(2)}, \cdots, \Delta_{p}^{(2)}\right]^{\prime}$, satisfies linear equations $A \Delta=b$, where $p \times p$ matrix $A$ and $p$-vector $b$ are given by

$$
\begin{aligned}
& A=\left(\begin{array}{cccc}
(n+p-1)(n+p+1) & n+p-3 & \cdots \cdots & n-p+1 \\
n+p-3 & (n+p-3)(n+p-1) & \cdots \cdots & n-p+1 \\
\cdots \cdots & \cdots \cdots & \cdots \cdots & \cdots \cdots \\
n-p+1 & n-p+1 & \cdots \cdots & (n-p+1)(n-p+3)
\end{array}\right) \\
& b=(n+p-1, n+p-3, \cdots, n-p+1)^{\prime} .
\end{aligned}
$$

With this $A$, the risk is given by

$$
R_{2}\left(\hat{\Sigma}_{J S}^{(2)}, \Sigma^{\prime}\right)=\frac{1}{2} p-\frac{1}{2} \sum_{j=1}^{p}(n-2 j+p+1) \Delta_{j}^{(2)} .
$$

We can see by checking the exact values of $\Delta^{(1)}$ and $\Delta^{(2)}$ that the choice of $\Delta_{j}^{(1)}$ is always larger than $\Delta_{j}^{(2)}$ and the risks of $\hat{\Sigma}_{J S}^{(1)}$ are larger than that of $\hat{\Sigma}_{J S}^{(2)}$. The best scalar multiple $1 / n$ for $L_{1}$ loss and $1 /(n+p+1)$ for $L_{2}$ loss lie always smaller than the middle of $\Delta_{1}, \cdots, \Delta_{p}$. Sharma [13] gives the values of $R_{2}\left(\hat{\Sigma}_{J S}^{(2)}, \Sigma\right)$ for $p=2$ and $n=5(5) 30$. Using (3.13), we can evaluate $\Delta$ for large $n$, giving

$$
\begin{aligned}
\Delta_{j}^{(2)}=\frac{1}{n}-\frac{2}{n^{2}}(p+1-j)+\frac{1}{n^{3}}\left[4(p+1)^{2}-(8 p+9) j+5 j^{2}\right] \\
+\frac{1}{3 n^{4}}\left[-2(p+1)\left(11 p^{2}+22 p+12\right)+\left(66 p^{2}+150 p+85\right) j\right. \\
\left.-3(28 p+33) j^{2}+38 j^{3}\right]+O\left(n^{-5}\right)
\end{aligned}
$$

and

$$
R_{2}\left(\hat{\Sigma}_{J S}^{(2)}, \nu^{\prime}\right)=\frac{p(p+1)}{2 n}-\frac{p(p+1)(2 p+1)}{3 n^{2}}+\frac{p^{2}(p+1)^{2}}{n^{3}}+O\left(n^{-4}\right)
$$

Note that optimal scalar multiplier for $S$ is $1 / n$ under $L_{1}$ loss and $1 /(n+p+1)$ under $L_{2}$ loss. Asymptotic expansion of $\Delta_{j}^{(1)}=1 /(n+p+1-2 j)$ replaced $n$ by $n+p+1$ yields the same terms as in (3.15) up to order $n^{-2}$. The difference of the risks, $R_{2}\left(\hat{\Sigma}_{J S}^{(2)}, \Sigma\right)-R_{2}\left(\hat{\Sigma}_{O}^{(2)}, \Sigma\right)$ in the asymptotic form is exactly the same as (3.12) up to $O\left(n^{-2}\right)$. In Table 3 , exact and asymptotic values of $R_{2}\left(\hat{\Sigma}_{J S}^{(2)}, \Sigma\right)$ are shown based on (3.14) and (3.16). We can see that the asymptotic approximations are worse than $R_{1}\left(\hat{\Sigma}_{J S}^{(1)}, \Sigma\right)$ and are comparative for $R_{2}\left(\hat{\Sigma}_{O}^{(2)}, \Sigma\right)$. This suggests that the loss $L_{1}$ is favourable for the asymptotic approximations. The maximum rate of reduction of risks for $\hat{\Sigma}_{J S}^{(1)}$ with respect to $\hat{\Sigma}_{o}^{(1)}$ within Tables 1 and 2 is given by $17 \%$ for $n=8$ and $p=6$. However the corresponding rate for $L_{2}$ loss in Table 3 is only $5 \%$. 
Table 3. Exact and asymptotic values of $R_{2}\left(\hat{\Sigma}_{J S}^{(2)}, \Sigma^{\prime}\right)$

\begin{tabular}{|c|c|c|c|c|c|c|}
\hline & & $n=8$ & $n=16$ & $n=32$ & $n=64$ & $n=128$ \\
\hline$p=2$ & $\begin{array}{l}O\left(n^{-1}\right) \\
O\left(n^{-2}\right) \\
O\left(n^{-3}\right)\end{array}$ & $\begin{array}{r}.37500 \\
-.15625 \\
.07031\end{array}$ & $\begin{array}{r}.18750 \\
-.03906 \\
.00879\end{array}$ & $\begin{array}{r}.093750 \\
-.009766 \\
.001099\end{array}$ & $\begin{array}{r}.046875 \\
-.002441 \\
.000137\end{array}$ & $\begin{array}{r}.023438 \\
-.000610 \\
.000017\end{array}$ \\
\hline & $\underset{\text { exact }}{\text { approx. }}$ & $\begin{array}{l}.289 \\
.26697\end{array}$ & $\begin{array}{l}.1572 \\
.15559\end{array}$ & $\begin{array}{l}.0851 \\
.084970\end{array}$ & $\begin{array}{l}.04457 \\
.044563\end{array}$ & $\begin{array}{l}.022844 \\
.022844\end{array}$ \\
\hline$p=3$ & $\begin{array}{l}O\left(n^{-1}\right) \\
O\left(n^{-2}\right) \\
O\left(n^{-3}\right)\end{array}$ & $\begin{array}{r}.75000 \\
-.43750 \\
.28125\end{array}$ & $\begin{array}{r}.37500 \\
-.10938 \\
.03516\end{array}$ & $\begin{array}{r}.18750 \\
-.02734 \\
.00440\end{array}$ & $\begin{array}{r}.093750 \\
-.006836 \\
.000549\end{array}$ & $\begin{array}{r}.046875 \\
-.001709 \\
.000069\end{array}$ \\
\hline & $\begin{array}{r}\text { approx. } \\
\text { exact }\end{array}$ & $\begin{array}{l}.59 \\
.48250\end{array}$ & $\begin{array}{l}.301 \\
.29211\end{array}$ & $\begin{array}{l}.1646 \\
.16393\end{array}$ & $\begin{array}{l}.08746 \\
.087422\end{array}$ & $\begin{array}{l}.045235 \\
.045232\end{array}$ \\
\hline$p=4$ & $\begin{array}{l}O\left(n^{-1}\right) \\
O\left(n^{-2}\right) \\
O\left(n^{-3}\right)\end{array}$ & $\begin{array}{r}1.2500 \\
-.9375 \\
.7813\end{array}$ & $\begin{array}{r}.62500 \\
-.23438 \\
.09766\end{array}$ & $\begin{array}{r}.31250 \\
-.05859 \\
.01221\end{array}$ & $\begin{array}{r}.15625 \\
-.01465 \\
.00153\end{array}$ & $\begin{array}{r}.078125 \\
-.003662 \\
.000191\end{array}$ \\
\hline & $\underset{\text { exact }}{\text { approx. }}$ & $\begin{array}{l}1.09 \\
.73548\end{array}$ & $\begin{array}{l}.488 \\
.45918\end{array}$ & $\begin{array}{l}.266 \\
.26397\end{array}$ & $\begin{array}{l}.1431 \\
.14298\end{array}$ & $\begin{array}{l}.07465 \\
.074644\end{array}$ \\
\hline$p=5$ & $\begin{array}{l}O\left(n^{-1}\right) \\
O\left(n^{-2}\right) \\
O\left(n^{-3}\right)\end{array}$ & $\begin{array}{r}1.8750 \\
-1.7188 \\
1.7578\end{array}$ & $\begin{array}{r}.9375 \\
-.4297 \\
.2197\end{array}$ & $\begin{array}{r}.46875 \\
-.10742 \\
.02747\end{array}$ & $\begin{array}{r}.23438 \\
-.02686 \\
.00343\end{array}$ & $\begin{array}{r}.117188 \\
-.006714 \\
.000429\end{array}$ \\
\hline & $\begin{array}{r}\text { approx. } \\
\text { exact }\end{array}$ & $\begin{array}{l}1.9 \\
1.0189\end{array}$ & $\begin{array}{l}.73 \\
.65233\end{array}$ & $\begin{array}{l}.389 \\
.38311\end{array}$ & $\begin{array}{l}.2110 \\
.21056\end{array}$ & $\begin{array}{l}.11090 \\
.11088\end{array}$ \\
\hline$p=6$ & $\begin{array}{l}O\left(n^{-1}\right) \\
O\left(n^{-2}\right) \\
O\left(n^{-3}\right)\end{array}$ & $\begin{array}{r}2.625 \\
-2.844 \\
3.445\end{array}$ & $\begin{array}{r}1.3125 \\
-.7109 \\
.4307\end{array}$ & $\begin{array}{r}.65625 \\
-.17773 \\
.05383\end{array}$ & $\begin{array}{r}.32813 \\
-.04443 \\
.00673\end{array}$ & $\begin{array}{r}.164063 \\
-.011108 \\
.000841\end{array}$ \\
\hline & $\begin{array}{r}\text { approx. } \\
\text { exact }\end{array}$ & $\begin{array}{l}3.2 \\
1.3283\end{array}$ & $\begin{array}{l}1.03 \\
.86807\end{array}$ & $\begin{array}{l}.532 \\
.51965\end{array}$ & $\begin{array}{l}.2904 \\
.28952\end{array}$ & $\begin{array}{l}.15380 \\
.15374\end{array}$ \\
\hline
\end{tabular}

\section{Risks under $L_{1}$ loss}

4.1. Risk of Haff's estimator. As Sharma [13] noted, the exact values of the risks of Haff's estimators are difficult to compute. Asymptotic evaluation of them gives some useful information. We shall put $C=I$ in (1.3) without loss of generality and assume that $t(u)=b=$ constant, namely, the estimator

$$
\hat{\Sigma}_{H}^{(1)}=\frac{1}{n}\left(S+\frac{b}{\operatorname{tr} S^{-1}} I\right)
$$

is considered for $L_{1}$ loss. The difference of risks can be written by

$$
\begin{aligned}
& R_{1}\left(\hat{\Sigma}_{H}^{(1)}, \Sigma^{\prime}\right)-R_{1}\left(\hat{\Sigma}_{O}^{(1)}, \Sigma\right) \\
& \quad=\frac{b}{n} E\left[\frac{\operatorname{tr} \Sigma^{\prime-1}}{\operatorname{tr} S^{-1}}\right]-E\left[\log \left|I+\frac{b}{\operatorname{tr} S^{-1}} S^{-1}\right|\right],
\end{aligned}
$$

which is bounded from above by

$$
\frac{b}{n} E\left[\frac{\operatorname{tr} \Sigma^{-1}}{\operatorname{tr} S^{-1}}\right]-b+\frac{b^{2}}{2} E\left[\frac{\operatorname{tr} S^{-2}}{\left(\operatorname{tr} S^{-1}\right)^{2}}\right] \text {. }
$$

By the Wishart identity due to Haff [5], we get 


$$
E\left[\frac{\operatorname{tr} \Sigma^{-1}}{\operatorname{tr} S^{-1}}\right]=n-p-1+2 E\left[\frac{\operatorname{tr} S^{-2}}{\left(\operatorname{tr} S^{-1}\right)^{2}}\right]
$$

This yields an upper bound of (4.2)

$$
\frac{b}{n}\left(-p-1+2+\frac{n b}{2}\right)
$$

which is negative if and only if $0 \leqq b \leqq 2(p-1) / n$, and the minimum value is attained by $b=(p-1) / n$. This is the special case of Theorem 4.3 by Haff [5]. We impose this restriction on $b$. Note that $b=O\left(n^{-1}\right)$ and $Y=\sqrt{n}(S / n-\Sigma)$ converges in law to a $p(p+1) / 2$ variate normal distribution with mean zero. We can evaluate (4.2) asymptotically as

$$
\frac{b}{n}\left\{E\left[\frac{\operatorname{tr} \Sigma^{-1}}{\operatorname{tr} S^{-1}}\right]-n+\frac{n b}{2} E\left[\frac{\operatorname{tr} S^{-2}}{\left(\operatorname{tr} S^{-1}\right)^{2}}\right]-\frac{b^{2} n}{3} \frac{\operatorname{tr} \Sigma^{-3}}{\left(\operatorname{tr} \Sigma^{-1}\right)^{3}}\right\}+O\left(n^{-4}\right) .
$$

In getting the last term of (4.6), we should take $E\left[\operatorname{tr} S^{-3} /\left(\operatorname{tr} S^{-1}\right)^{3}\right]$, which can be evaluated by writing $S / n=\Sigma+Y / \sqrt{n}$ and noting that $E(Y)=0$ and $Y=O_{p}(1)$, giving $\operatorname{tr} \Sigma^{-3} /\left(\operatorname{tr} \Sigma^{-1}\right)^{3}+O\left(n^{-1}\right)$. Now we need the following lemma to complete our asymptotic expansion.

Lemma 4.1. Let $S$ have a Wishart distribution $W_{p}(n, \Sigma)$. Then

$$
\begin{aligned}
E\left[\frac{\operatorname{tr} S^{-2}}{\left(\operatorname{tr} S^{-1}\right)^{2}}\right]= & \frac{\operatorname{tr} \Sigma^{-2}}{\left(\operatorname{tr} \Sigma^{-1}\right)^{2}}+\frac{1}{n}\left\{6 \frac{\left(\operatorname{tr} \Sigma^{-2}\right)^{2}}{\left(\operatorname{tr} \Sigma^{-1}\right)^{4}}\right. \\
& \left.-8 \frac{\operatorname{tr} \Sigma^{-3}}{\left(\operatorname{tr} \Sigma^{-1}\right)^{3}}+\frac{\operatorname{tr} \Sigma^{-2}}{\left(\operatorname{tr} \Sigma^{-1}\right)^{2}}+1\right\}+O\left(n^{-2}\right) .
\end{aligned}
$$

Proof. From the Wishart identity, we get

$$
\begin{aligned}
E\left[\frac{\operatorname{tr} S^{-2}}{\left(\operatorname{tr} S^{-1}\right)^{2}} \operatorname{tr} \Sigma^{-1}\right]= & 4 E\left[\frac{\left(\operatorname{tr} S^{-2}\right)^{2}}{\left(\operatorname{tr} S^{-1}\right)^{3}}-\frac{\operatorname{tr} S^{-3}}{\left(\operatorname{tr} S^{-1}\right)^{2}}\right] \\
& +(n-p-1) E\left[\frac{\operatorname{tr} S^{-2}}{\operatorname{tr} S^{-1}}\right] \\
E\left[\frac{\operatorname{tr} S^{-2}}{\operatorname{tr} S^{-1}} \operatorname{tr} \Sigma^{-1}\right]= & 2 E\left[\frac{\left(\operatorname{tr} S^{-2}\right)^{2}}{\left(\operatorname{tr} S^{-1}\right)^{2}}-2 \frac{\operatorname{tr} S^{-3}}{\operatorname{tr} S^{-1}}\right] \\
+ & (n-p-1) E\left[\operatorname{tr} S^{-2}\right] .
\end{aligned}
$$

By Haff [4], we know that

$$
\begin{aligned}
E\left[\operatorname{tr} S^{-2}\right] & =\frac{\left(\operatorname{tr} \Sigma^{-1}\right)^{2}}{(n-p)(n-p-1)(n-p-3)}+\frac{\operatorname{tr} \Sigma^{-2}}{(n-p)(n-p-3)} \\
& =\frac{1}{n^{2}} \operatorname{tr} \Sigma^{-2}+\frac{2 p+3}{n^{3}} \operatorname{tr} \Sigma^{-2}+\frac{1}{n^{3}}\left(\operatorname{tr} \Sigma^{-1}\right)^{2}+O\left(n^{-4}\right)
\end{aligned}
$$


Combined with these formulas, we get the desired result (4.7). Substituting (4.4) and (4.7) into (4.6) and using (3.12) we get

THEOREM 4.1. An asymptotic expansion of the difference of risks between Haff's estimator $\hat{\Sigma}_{H}^{(1)}$ defined by (4.1) with $b=(p-1) / n$ and James and Stein's minimax estimator $\hat{\Sigma}_{J S}^{(1)}$ for $L_{1}$ loss is given by

$$
\begin{aligned}
& R_{1}\left(\hat{\Sigma}_{H}^{(1)}, \Sigma\right)-R_{1}\left(\hat{\Sigma}_{J S}^{(1)}, \Sigma^{\prime}\right)=\frac{p-1}{6 n^{2}}\left\{(p+1)(p-6)+3(p+3) \frac{\operatorname{tr} \Sigma^{-2}}{\left(\operatorname{tr} \Sigma^{-1}\right)^{2}}\right\} \\
& +\frac{(p-1)(p+3)}{2 n^{3}}\left\{6 \frac{\left(\operatorname{tr} \Sigma^{-2}\right)^{2}}{\left(\operatorname{tr} \Sigma^{-1}\right)^{4}}-8 \frac{\operatorname{tr} \Sigma^{-3}}{\left(\operatorname{tr} \Sigma^{-1}\right)^{3}}+\frac{\operatorname{tr} \Sigma^{-2}}{\left(\operatorname{tr} \Sigma^{-1}\right)^{2}}+1\right\} \\
& -\frac{(p-1)^{3}}{3 n^{3}} \frac{\operatorname{tr} \Sigma^{-3}}{\left(\operatorname{tr} \Sigma^{-1}\right)^{3}}+O\left(n^{-4}\right) .
\end{aligned}
$$

We can see that the term of $O\left(n^{-2}\right)$ in (4.11) is always positive, if $p \geqq 6$. This shows that the risk of $\hat{\Sigma}_{H}^{(1)}$ is always larger than that of $\hat{\Sigma}_{J S}^{(1)}$ asymptotically, if $p \geqq 6$. Note that

$$
\frac{1}{p} \leqq \frac{\operatorname{tr} \Sigma^{-2}}{\left(\operatorname{tr} \Sigma^{-1}\right)^{2}} \leqq 1
$$

The lower and upper bounds of $O\left(n^{-2}\right)$ in (4.11) are given by

$$
\frac{1}{6}(p-1)\left(p^{2}-5 p-3+\frac{9}{p}\right) \text { and } \frac{1}{6}(p-1)\left(p^{2}-2 p+3\right)
$$

Some numerical values are given in the following:

$$
\begin{array}{clccc}
p=2 & \multicolumn{5}{c}{\text { Ranges of } O\left(n^{-2}\right) \text { in } \frac{(4.11) .}{p=5}} & p=6 \\
\left(-\frac{3}{4}, \frac{1}{2}\right) & (-2,2) & \left(-\frac{19}{8}, \frac{11}{2}\right) & \left(-\frac{4}{5}, 12\right) & \left(\frac{15}{4}, \frac{45}{2}\right)
\end{array}
$$

The risk is unchanged for any scalar multiple of $\Sigma$. Some numerical values based on (4.11) are given in Table 4. The term of $O\left(n^{-3}\right)$ gives some idea for the error of our asymptotic approximation. For $\Sigma^{-1}=\lambda \operatorname{diag}(1,1, \cdots, 1)$, the lower bound of (4.12) is attained and for $\Sigma^{-1} \rightarrow \lambda \operatorname{diag}(1,0, \cdots, 0)$, the upper bound is approached. In Table 4 we write $\Sigma^{-1}=\lambda(1, \cdots, 1)$ instead of $\Sigma^{-1}=\lambda \operatorname{diag}(1, \cdots, 1)$ for abbreviation. Inspection of Table 4 shows that for $p \geqq 6$, the risk differences are positive and that for $p=5$ and $\Sigma^{-1}=\lambda \operatorname{diag}(1, \cdots, 1)$, the values are positive for $n=8$ and $n=16$, while they are negative for $n \geqq 32$. Precisely speaking they are positive for $n \leqq 21$ and negative for $n \geqq 22$. Whether this is due to the poor accuracy of the asymptotic approximation for small $n$ is not clear. For $p \leqq 4$ and $\Sigma^{-1}=\lambda \operatorname{diag}(1, \cdots, 1)$, the values are all negative. Thus $p=5$ is the boundary. $\hat{\Sigma}_{H}^{(1)}$ is better than $\hat{\Sigma}_{J S}^{(1)}$ for these type of $\Sigma$ if $p \leqq 5$. For $0 \leqq b \leqq 2(p-1) / n$, inequality $R_{1}\left(\hat{\Sigma}_{H}^{(1)}, \Sigma\right)<R_{1}\left(\hat{\Sigma}_{o}^{(1)}, \Sigma\right)$ holds 
exactly. This can be verified also by the asymptotic consideration, namely, we have

$$
\begin{aligned}
& R_{1}\left(\hat{\Sigma}_{H}^{(1)}, \Sigma^{\prime}\right)-R_{1}\left(\hat{\Sigma}_{O}^{(1)}, \nu^{\prime}\right) \\
& \quad=\frac{p-1}{n^{2}}\left[-(p+1)+\frac{1}{2}(p+3) \frac{\operatorname{tr} \Sigma^{-2}}{\left(\operatorname{tr} \Sigma^{-1}\right)^{2}}\right]+O\left(n^{-3}\right) .
\end{aligned}
$$

The term of $O\left(n^{-2}\right)$ is always negative because of (4.12). This gives again a weak support as in (3.12) for the usefulness of the asymptotic comparison, when exact inequality between risks is not known. From Tables 1 and 4, we can compute the rates of the reduction of the risks of Haff's estimator $\hat{\Sigma}_{H}^{(1)}$ with respect to the

\begin{tabular}{|c|c|c|c|c|c|c|}
\hline$\Sigma^{-1}$ & & $n=8$ & $n=16$ & $n=32$ & $n=64$ & $n=128$ \\
\hline$p=2$ & $\begin{array}{l}O\left(n^{-2}\right) \\
O\left(n^{-3}\right) \\
\text { approx. }\end{array}$ & $\begin{array}{c}-.011719 \\
-.004720 \\
-.0070\end{array}$ & $\begin{array}{r}-.002930 \\
-.000590 \\
-.00234\end{array}$ & $\begin{array}{r}-.000732 \\
-.000074 \\
-.000659\end{array}$ & $\begin{array}{r}-.000183 \\
-.000009 \\
-.000174\end{array}$ & $\begin{array}{r}-.000046 \\
-.000001 \\
-.000045\end{array}$ \\
\hline$\lambda(1,2)$ & $\begin{array}{l}O\left(n^{-2}\right) \\
O\left(n^{-3}\right) \\
\text { approx. }\end{array}$ & $\begin{array}{c}-.009549 \\
-.003400 \\
-.0061\end{array}$ & $\begin{array}{c}-.002387 \\
-.000425 \\
-.00196\end{array}$ & $\begin{array}{r}-.000597 \\
.000053 \\
-.000544\end{array}$ & $\begin{array}{r}-.000149 \\
.000007 \\
-.000143\end{array}$ & $\begin{array}{r}-.000037 \\
-.000001 \\
-.000036\end{array}$ \\
\hline$\lambda(1,10)$ & $\begin{array}{l}O\left(n^{-2}\right) \\
O\left(n^{-3}\right) \\
\text { approx. }\end{array}$ & $\begin{array}{c}.001356 \\
-.000496 \\
.00086\end{array}$ & $\begin{array}{r}.000339 \\
-.000062 \\
.000277\end{array}$ & $\begin{array}{r}.000085 \\
-.000008 \\
.000077\end{array}$ & $\begin{array}{r}.000021 \\
-.000001 \\
.000020\end{array}$ & $\begin{array}{r}.000005 \\
-.000000 \\
.000005\end{array}$ \\
\hline$\lambda(1,0)$ & $\begin{array}{l}O\left(n^{-2}\right) \\
O\left(n^{-3}\right) \\
\text { approx. }\end{array}$ & $\begin{array}{c}.007813 \\
-.000651 \\
.00716\end{array}$ & $\begin{array}{r}.001953 \\
-.000081 \\
.001872\end{array}$ & $\begin{array}{r}.000488 \\
-.000010 \\
.000478\end{array}$ & $\begin{array}{r}.000122 \\
-.000001 \\
.000121\end{array}$ & $\begin{array}{r}.000031 \\
-.000000 \\
.000030\end{array}$ \\
\hline$\lambda(1,1,1)$ & $\begin{array}{l}O\left(n^{-2}\right) \\
O\left(n^{-3}\right) \\
\text { approx. }\end{array}$ & $\begin{array}{c}-.031250 \\
-.012442 \\
-.019\end{array}$ & $\begin{array}{c}-.007813 \\
-.001555 \\
-.0063\end{array}$ & $\begin{array}{c}-.001953 \\
-.000194 \\
-.00176\end{array}$ & $\begin{array}{r}-.000488 \\
-.000024 \\
-.000464\end{array}$ & $\begin{array}{r}-.000122 \\
.000003 \\
-.000119\end{array}$ \\
\hline$\lambda(1,2,3)$ & $\begin{array}{l}O\left(n^{-2}\right) \\
O\left(n^{-3}\right) \\
\text { approx. }\end{array}$ & $\begin{array}{c}-.026042 \\
-.010417 \\
-.016\end{array}$ & $\begin{array}{c}-.006510 \\
-.001302 \\
-.0052\end{array}$ & $\begin{array}{c}-.001628 \\
-.000163 \\
-.00146\end{array}$ & $\begin{array}{r}-.000407 \\
-.000020 \\
-.000387\end{array}$ & $\begin{array}{r}-.000102 \\
.000003 \\
-.000099\end{array}$ \\
\hline$\lambda\left(1,10,10^{2}\right)$ & $\begin{array}{l}O\left(n^{-2}\right) \\
O\left(n^{-3}\right) \\
\text { approx. }\end{array}$ & $\begin{array}{c}.014358 \\
-.003847 \\
.0105\end{array}$ & $\begin{array}{r}.003590 \\
-.000481 \\
.00311\end{array}$ & $\begin{array}{r}.000897 \\
-.000060 \\
.000837\end{array}$ & $\begin{array}{r}.000224 \\
-.000008 \\
.000217\end{array}$ & $\begin{array}{r}.000056 \\
-.000001 \\
.000055\end{array}$ \\
\hline$\lambda(1,0,0)$ & $\begin{array}{l}O\left(n^{-2}\right) \\
O\left(n^{-3}\right) \\
\text { approx. }\end{array}$ & $\begin{array}{c}.031250 \\
-.005208 \\
.0260\end{array}$ & $\begin{array}{c}.007813 \\
-.000651 \\
.00716\end{array}$ & $\begin{array}{r}.001953 \\
-.000081 \\
.001872\end{array}$ & $\begin{array}{r}.000488 \\
-.000010 \\
.000478\end{array}$ & $\begin{array}{r}.000122 \\
-.000001 \\
.000121\end{array}$ \\
\hline$\lambda(1, \cdots, 1)$ & $\begin{array}{l}O\left(n^{-2}\right) \\
O\left(n^{-3}\right) \\
\text { approx. }\end{array}$ & $\begin{array}{l}-.037109 \\
.021973 \\
-.015\end{array}$ & $\begin{array}{c}-.009277 \\
.002747 \\
-.0065\end{array}$ & $\begin{array}{c}-.002319 \\
.000343 \\
-.00198\end{array}$ & $\begin{array}{r}-.000580 \\
.000043 \\
-.000537\end{array}$ & $\begin{array}{r}-.000145 \\
.000005 \\
-.000140\end{array}$ \\
\hline$\lambda(1,2,3,4)$ & $\begin{array}{l}O\left(n^{-2}\right) \\
O\left(n^{-3}\right) \\
\text { approx. }\end{array}$ & $\begin{array}{c}-.028906 \\
-.019570 \\
-.009\end{array}$ & $\begin{array}{c}-.007227 \\
-.002446 \\
-.0048\end{array}$ & $\begin{array}{c}-.001807 \\
-.000306 \\
-.00150\end{array}$ & $\begin{array}{r}-.000452 \\
-.000038 \\
-.000413\end{array}$ & $\begin{array}{r}-.000113 \\
.000005 \\
-.000108\end{array}$ \\
\hline$\left(1,10,10^{2}, 10^{3}\right)$ & $\begin{array}{l}O\left(n^{-2}\right) \\
O\left(n^{-3}\right) \\
\text { approx. }\end{array}$ & $\begin{array}{l}.056135 \\
-.012895 \\
.043\end{array}$ & $\begin{array}{l}.014034 \\
-.001612 \\
.0124\end{array}$ & $\begin{array}{c}.003508 \\
-.000201 \\
.00331\end{array}$ & $\begin{array}{r}.000877 \\
-.000025 \\
.000852\end{array}$ & $\begin{array}{r}.000219 \\
-.000003 \\
.000216\end{array}$ \\
\hline$\lambda(1,0,0,0)$ & $\begin{array}{l}O\left(n^{-2}\right) \\
O\left(n^{-3}\right) \\
\text { approx. }\end{array}$ & $\begin{array}{l}.085938 \\
-.017578 \\
.068\end{array}$ & $\begin{array}{l}.021484 \\
-.002197 \\
.0193\end{array}$ & $\begin{array}{c}.005371 \\
-.000275 \\
.00510\end{array}$ & $\begin{array}{r}.001343 \\
-.000034 \\
.001308\end{array}$ & $\begin{array}{r}.000336 \\
-.000004 \\
.000331\end{array}$ \\
\hline
\end{tabular}

Table 4. Asymptotic values of $R_{1}\left(\hat{\Sigma}_{H}^{(1)}, \Sigma\right)-R_{1}\left(\hat{\Sigma}_{J S}^{(1)}, \Sigma\right)$ 
Table 4. (continued)

\begin{tabular}{|c|c|c|c|c|c|c|}
\hline$\Sigma^{-1}$ & & $n=8$ & $n=16$ & $n=32$ & $n=64$ & $n=128$ \\
\hline$\lambda(1, \cdots, 1)$ & $\begin{array}{l}O\left(n^{-2}\right) \\
O\left(n^{-3}\right) \\
\text { approx. }\end{array}$ & $\begin{array}{c}-.012500 \\
.033333 \\
.021\end{array}$ & $\begin{array}{c}-.003125 \\
.004167 \\
.0010\end{array}$ & $\begin{array}{r}-.000781 \\
.000521 \\
-.00026\end{array}$ & $\begin{array}{r}-.000195 \\
-.000065 \\
-.000130\end{array}$ & $\begin{array}{r}-.000049 \\
-.000008 \\
-.000041\end{array}$ \\
\hline$\lambda(1,2, \cdots, 5)$ & $\begin{array}{l}O\left(n^{-2}\right) \\
O\left(n^{-3}\right) \\
\text { approx. }\end{array}$ & $\begin{array}{c}-.001389 \\
.030648 \\
.029\end{array}$ & $\begin{array}{c}-.000347 \\
.003831 \\
.0035\end{array}$ & $\begin{array}{r}-.000087 \\
.000479 \\
.00039\end{array}$ & $\begin{array}{r}-.000022 \\
.000060 \\
.000038\end{array}$ & $\begin{array}{r}-.000005 \\
.000007 \\
.000002\end{array}$ \\
\hline$\lambda\left(1,10, \cdots, 10^{4}\right)$ & $\begin{array}{l}O\left(n^{-2}\right) \\
O\left(n^{-3}\right) \\
\text { approx. }\end{array}$ & $\begin{array}{l}.142050 \\
-.030504 \\
.112\end{array}$ & $\begin{array}{c}.035512 \\
-.003813 \\
.0317\end{array}$ & $\begin{array}{r}.008878 \\
-.000477 \\
.00840\end{array}$ & $\begin{array}{r}.002220 \\
-.000060 \\
.002160\end{array}$ & $\begin{array}{r}.000555 \\
-.000007 \\
.000547\end{array}$ \\
\hline$\lambda(1,0, \cdots, 0)$ & $\begin{array}{l}O\left(n^{-2}\right) \\
O\left(n^{-3}\right) \\
\text { approx. }\end{array}$ & $\begin{array}{c}.187500 \\
-.041667 \\
.146\end{array}$ & $\begin{array}{c}.046875 \\
-.005208 \\
.0417\end{array}$ & $\begin{array}{r}.011719 \\
-.000651 \\
.01107\end{array}$ & $\begin{array}{r}.002930 \\
-.000081 \\
.002848\end{array}$ & $\begin{array}{r}.000732 \\
-.000010 \\
.000722\end{array}$ \\
\hline$\lambda(1, \cdots, 1)$ & $\begin{array}{l}O\left(n^{-2}\right) \\
O\left(n^{-3}\right) \\
\text { approx. }\end{array}$ & $\begin{array}{l}.058594 \\
.046568 \\
.105\end{array}$ & $\begin{array}{l}.014648 \\
.005821 \\
.0205\end{array}$ & $\begin{array}{l}.003662 \\
.000728 \\
.00439\end{array}$ & $\begin{array}{l}.000916 \\
.000091 \\
.001006\end{array}$ & $\begin{array}{l}.000229 \\
.000011 \\
.000240\end{array}$ \\
\hline$\lambda(1,2, \cdots, 6)$ & $\begin{array}{l}O\left(n^{-2}\right) \\
O\left(n^{-3}\right) \\
\text { approx. }\end{array}$ & $\begin{array}{l}.072545 \\
.043624 \\
.116\end{array}$ & $\begin{array}{l}.018136 \\
.005453 \\
.0236\end{array}$ & $\begin{array}{l}.004534 \\
.000682 \\
.00522\end{array}$ & $\begin{array}{l}.001134 \\
.000085 \\
.001219\end{array}$ & $\begin{array}{l}.000283 \\
.000011 \\
.000294\end{array}$ \\
\hline$\lambda\left(1,10, \cdots, 10^{5}\right)$ & $\begin{array}{l}O\left(n^{-2}\right) \\
O\left(n^{-3}\right) \\
\text { approx. }\end{array}$ & $\begin{array}{c}.287643 \\
-.059523 \\
.228\end{array}$ & $\begin{array}{c}.071911 \\
-.007440 \\
.0645\end{array}$ & $\begin{array}{r}.017978 \\
-.000930 \\
.01705\end{array}$ & $\begin{array}{r}.004494 \\
-.000116 \\
.00438\end{array}$ & $\begin{array}{r}.001124 \\
-.000015 \\
.001109\end{array}$ \\
\hline$\lambda(1,0, \cdots, 0)$ & $\begin{array}{l}O\left(n^{-2}\right) \\
O\left(n^{-3}\right) \\
\text { approx. }\end{array}$ & $\begin{array}{l}.351563 \\
-.081380 \\
.270\end{array}$ & $\begin{array}{l}.087891 \\
-.010173 \\
.078\end{array}$ & $\begin{array}{c}.021973 \\
-.001272 \\
.0207\end{array}$ & $\begin{array}{r}.005493 \\
-.000159 \\
.00533\end{array}$ & $\begin{array}{r}.001373 \\
-.000020 \\
.001353\end{array}$ \\
\hline
\end{tabular}

maximum likelihood estimator $\hat{\Sigma}_{O}^{(1)}$, namely $100 \times\left\{R_{1}\left(\hat{\Sigma}_{O}^{(1)}, \Sigma^{\prime}\right)-R_{1}\left(\hat{\Sigma}_{H}^{(1)}, \Sigma\right)\right\} / R_{1}\left(\hat{\Sigma}_{O}^{(1)}, \Sigma^{\prime}\right)$, which range above to $8 \%$ for $n \geqq 16$. The rates of the reduction of the risks of $\hat{\Sigma}_{H}^{(1)}$ with respect to $\hat{\Sigma}_{J S}^{(1)}$ range only from $-5.6 \%$ to $1.6 \%$ for $n \geqq 16$ in Table 4 .

4.2. Risk of new estimator. Now we shall consider the risk of a new estimator $\hat{\Sigma}^{(1)}$ given in (1.6), We can write the risk difference

$$
\begin{aligned}
& R_{1}\left(\hat{\Sigma}^{(1)}, \Sigma^{\prime}\right)-R_{1}\left(\hat{\Sigma}_{O}^{(1)}, \Sigma^{\prime}\right) \\
& \quad=\frac{b}{n}\left(\operatorname{tr} \Sigma^{-1}\right) E\left[\frac{\operatorname{tr} S^{-1}}{\operatorname{tr} S^{-2}}\right]-E\left[\log \left|I+\frac{b \operatorname{tr} S^{-1}}{\operatorname{tr} S^{-2}} S^{-1}\right|\right] .
\end{aligned}
$$

By the Wishart identity, we get

$$
\begin{aligned}
E\left[\frac{\operatorname{tr} S^{-1}}{\operatorname{tr} S^{-2}} \operatorname{tr} \Sigma^{-1}\right]= & 4 E\left[\frac{\operatorname{tr} S^{-3} \operatorname{tr} S^{-1}}{\left(\operatorname{tr} S^{-2}\right)^{2}}\right]-2 \\
& +(n-p-1) E\left[\frac{\left(\operatorname{tr} S^{-1}\right)^{2}}{\operatorname{tr} S^{-2}}\right] .
\end{aligned}
$$

Using (4.16), the risk difference is bounded from above by

$$
\frac{b}{n}\left\{4 E\left[\frac{\operatorname{tr} S^{-3} \operatorname{tr} S^{-1}}{\left(\operatorname{tr} S^{-2}\right)^{2}}\right]-2+\left(\frac{b n}{2}-p-1\right) E\left[\frac{\left(\operatorname{tr} S^{-1}\right)^{2}}{\operatorname{tr} S^{-2}}\right]\right\} \text {. }
$$


Note that

$$
2 \frac{\operatorname{tr} S^{-3} \operatorname{tr} S^{-1}}{\left(\operatorname{tr} S^{-2}\right)^{2}} \leqq 1+\frac{\left(\operatorname{tr} S^{-1}\right)^{2}}{\operatorname{tr} S^{-2}}
$$

where the equality holds if and only if $S^{-1}=\lambda \operatorname{diag}(1,0, \cdots, 0)$ except for permutation of the diagonal elements. The upper bound (4.17) is further simplified as

$$
\frac{b}{n}\left(\frac{b n}{2}-p+1\right) E\left[\frac{\left(\operatorname{tr} S^{-1}\right)^{2}}{\operatorname{tr} S^{-2}}\right]
$$

Hence $\hat{\Sigma}^{(1)}$ dominates $\hat{\Sigma}_{0}^{(1)}$ if $0 \leqq b \leqq 2(p-1) / n$ and the minimum of (4.19) is attained by $b=(p-1) / n$. The choice of $b$ is the same as for the Haff's estimator.

To get asymptotic expansion of the risk difference (4.15), we can rewrite it as in (4.6) by

$$
\begin{gathered}
\frac{b}{n}\left\{\left(\frac{n b}{2}-p-1\right) E\left[\frac{\left(\operatorname{tr} S^{-1}\right)^{2}}{\operatorname{tr} S^{-2}}\right]-2+4 E\left[\frac{\operatorname{tr} S^{-3} \operatorname{tr} S^{-1}}{\left(\operatorname{tr} S^{-2}\right)^{2}}\right]\right\} \\
-\frac{b^{3}}{3} \frac{\left(\operatorname{tr} \Sigma^{-1}\right)^{3} \operatorname{tr} \Sigma^{-3}}{\left(\operatorname{tr} \Sigma^{-2}\right)^{3}}+O\left(n^{-4}\right) .
\end{gathered}
$$

To evaluate each expectation asymptotically, we need the following lemma.

Lemma 4.2. Let $S$ have a Wishart distribution $W_{p}(n, \Sigma)$. Then

$$
\begin{aligned}
& E\left[\frac{\left(\operatorname{tr} S^{-1}\right)^{2}}{\operatorname{tr} S^{-2}}\right] \\
& =\frac{\left(\operatorname{tr} \Sigma^{-1}\right)^{2}}{\operatorname{tr} \Sigma^{-2}}+\frac{1}{n}\left[8 \frac{\operatorname{tr} \Sigma^{-4}\left(\operatorname{tr} \Sigma^{-1}\right)^{2}}{\left(\operatorname{tr} \Sigma^{-2}\right)^{3}}-\frac{\left(\operatorname{tr} \Sigma^{-1}\right)^{4}}{\left(\operatorname{tr} \Sigma^{-2}\right)^{2}}\right. \\
& \left.\quad-8 \frac{\operatorname{tr} \Sigma^{-3} \operatorname{tr} \Sigma^{-1}}{\left(\operatorname{tr} \Sigma^{-2}\right)^{2}}-\frac{\left(\operatorname{tr} \Sigma^{-1}\right)^{2}}{\operatorname{tr} \Sigma^{-2}}+2\right]+O\left(n^{-2}\right), \\
& E\left[\frac{\operatorname{tr} S^{-1} \operatorname{tr} S^{-3}}{\left(\operatorname{tr} S^{-2}\right)^{2}}\right] \\
& =\frac{\operatorname{tr} \Sigma^{-1} \operatorname{tr} \Sigma^{-3}}{\left(\operatorname{tr} \Sigma^{-2}\right)^{2}}+\frac{1}{n}\left[24 \frac{\operatorname{tr} \Sigma^{-1} \operatorname{tr} \Sigma^{-3} \operatorname{tr} \Sigma^{-4}}{\left(\operatorname{tr} \Sigma^{-2}\right)^{4}}\right. \\
& \quad-\frac{2}{\left(\operatorname{tr} \Sigma^{-2}\right)^{3}}\left\{\left(\operatorname{tr} \Sigma^{-1}\right)^{3} \operatorname{tr} \Sigma^{-3}+12 \operatorname{tr} \Sigma^{-1} \operatorname{tr} \Sigma^{-5}\right. \\
& \left.\quad+4\left(\operatorname{tr} \Sigma^{-3}\right)^{2}\right\}+\frac{1}{\left(\operatorname{tr} \Sigma^{-2}\right)^{2}}\left\{\operatorname{tr} \Sigma^{-1} \operatorname{tr} \Sigma^{-3}+6 \operatorname{tr} \Sigma^{-4}\right\} \\
& \left.\quad+\frac{3\left(\operatorname{tr} \Sigma^{-1}\right)^{2}}{\operatorname{tr} \Sigma^{-2}}\right]+O\left(n^{-2}\right) .
\end{aligned}
$$


Unlike Lemma 4.1, it seems to be impossible to prove Lemma 4.2 from the Wishart identity only. We obtained it by another method used by Ito [6], Siotani [14], Okamoto [11], Sugiura [15], Fujikoshi [2] and others, that is, for analytic function $f(S)$, it holds

$$
E\left[f\left(\frac{1}{n} S\right)\right]=f(\Sigma)+\left.\frac{1}{n} \operatorname{tr}(\Sigma \partial)^{2} f(\Lambda)\right|_{\Lambda=\Sigma}+O\left(n^{-2}\right),
$$

where $\partial$ is a matrix of differential operators and its $(i, j)$ element is given by $(1 / 2)\left(1+\delta_{i j}\right)\left(\partial / \partial \lambda_{i j}\right)$ for $\Lambda=\left(\lambda_{i j}\right)$. The following lemma is useful for the repeated application of (4.23).

LeMmA 4.3. Let $E_{i j}(i \neq j)$ be $p \times p$ matrix having $1 / 2$ at the $(i, j)$ and $(j, i)$ positions and zero at other positions. Let $E_{i i}$ be diagonal matrix having 1 at $i$-th diagonal and zero otherwise. Then for any symmetric matrices $A=\left(a_{i j}\right)$ and $B=\left(b_{i j}\right)$,

$$
\sum_{i, j} \lambda_{i} \lambda_{j} \operatorname{tr} A E_{i j} \operatorname{tr} B E_{i j}=\sum_{i, j} \lambda_{i} \lambda_{j} a_{i j} b_{i j}
$$

$$
\sum_{i, j} \lambda_{i} \lambda_{j} \operatorname{tr} A E_{i j} B E_{i j}=\frac{1}{2} \sum_{i, j} \lambda_{i} \lambda_{j} a_{i j} b_{i j}+\frac{1}{2} \sum_{i} \lambda_{i} a_{i i} \sum_{j} \lambda_{j} b_{j j}
$$

Applying Lemma 4.2 to (4.20), we get

THEOREM 4.2. An asymptotic expansion of the difference of risks between new estimator $\hat{\Sigma}^{(1)}$ defined by (1.6) with $b=(p-1) / n$ and James and Stein's minimax estimator $\hat{\Sigma}_{J S}^{(1)}$ for $L_{1}$ loss is given by

$$
\begin{aligned}
& R_{1}\left(\hat{\Sigma}^{(1)}, \Sigma^{\prime}\right)-R_{1}\left(\hat{\Sigma}_{J S}^{(1)}, \Sigma^{\prime}\right)=\frac{p\left(p^{2}-1\right)}{6 n^{2}}+\frac{p-1}{n^{2}}\left[-2+4 \frac{\operatorname{tr} \Sigma^{-1} \operatorname{tr} \Sigma^{-3}}{\left(\operatorname{tr} \Sigma^{-2}\right)^{2}}\right. \\
& \left.-\frac{p+3}{2} \frac{\left(\operatorname{tr} \Sigma^{-1}\right)^{2}}{\operatorname{tr} \Sigma^{-2}}\right]+\frac{p-1}{n^{3}}\left[96 \frac{\operatorname{tr} \Sigma^{-1} \operatorname{tr} \Sigma^{-3} \operatorname{tr} \Sigma^{-4}}{\left(\operatorname{tr} \Sigma^{-2}\right)^{4}}\right. \\
& -\frac{1}{\left(\operatorname{tr} \Sigma^{-2}\right)^{3}}\left\{\left(8+\frac{(p-1)^{2}}{3}\right)\left(\operatorname{tr} \Sigma^{-1}\right)^{3} \operatorname{tr} \Sigma^{-3}+96 \operatorname{tr} \Sigma^{-1} \operatorname{tr} \Sigma^{-5}\right. \\
& \left.+32\left(\operatorname{tr} \Sigma^{-3}\right)^{2}+4(p+3)\left(\operatorname{tr} \Sigma^{-1}\right)^{2} \operatorname{tr} \Sigma^{-4}\right\} \\
& +\frac{1}{\left(\operatorname{tr} \Sigma^{-2}\right)^{2}}\left\{4(p+4) \operatorname{tr} \Sigma^{-1} \operatorname{tr} \Sigma^{-3}+24 \operatorname{tr} \Sigma^{-4}+\frac{p+3}{2}\left(\operatorname{tr} \Sigma^{-1}\right)^{4}\right\} \\
& \left.+\left(12+\frac{p+3}{2}\right) \frac{\left(\operatorname{tr} \Sigma^{-1}\right)^{2}}{\operatorname{tr} \Sigma^{-2}}-p-3\right]+O\left(n^{-4}\right)
\end{aligned}
$$

By the inequalities (4.12) and (4.18), the term of $O\left(n^{-2}\right)$ in (4.25) ranges from 


$$
-\frac{1}{3}(p-1)\left(p^{2}+4 p-6\right) \text { to } \frac{1}{6}(p-1)\left(p^{2}-2 p+3\right)
$$

The lower bound is obtained by noting that $\left(\operatorname{tr} \Sigma^{-1}\right)^{2} / \operatorname{tr} \Sigma^{-2} \leqq p$ and $\operatorname{tr} \Sigma^{-1} \operatorname{tr} \Sigma^{-3} /$ $\left(\operatorname{tr} \Sigma^{-2}\right)^{2} \geqq 1$, where both equalities are satisfied by $\Sigma^{-1}=\lambda I$. The upper bound is the same as for $\hat{\Sigma}_{H}^{(1)}$ given in (4.13), while the lower bound is smaller than that of $\hat{\Sigma}_{H}^{(1)}$, and is always negative. Some numerical values are given below. The lower bound is considerably smaller than (4.13).

$$
\begin{aligned}
& \text { Ranges of } O\left(n^{-2}\right) \text { in (4.25). } \\
& p=2 \quad p=3 \quad p=4 \quad p=5 \quad p=6 \\
& \left(-2, \frac{1}{2}\right) \quad(-10,2) \quad\left(-26, \frac{11}{2}\right) \quad(-52,12) \quad\left(-90, \frac{45}{2}\right)
\end{aligned}
$$

The upper bound is approached as $\Sigma^{-1} \rightarrow \lambda \operatorname{diag}(1,0, \cdots, 0)$ or any permutation of the diagonal elements of it. This shows that $\hat{\Sigma}^{(1)}$ is better than $\hat{\Sigma}_{J S}^{(1)}$ for $\Sigma^{-1}=\lambda I$ and worse for $\Sigma^{-1}=\lambda \operatorname{diag}(1,0, \cdots, 0)$, which is the same conclusion as in Haff's estimator $\hat{\Sigma}_{H}^{(1)}$. However the lower bound is always negative for $\hat{\Sigma}^{(1)}$ and it is not dominated by $\hat{\Sigma}_{J S}^{(1)}$ for any $p$ if $n$ is large.

Some numerical values based on Theorem 4.2 are given in Table 5 , in contrast to Table 4 . For $n=8$ and $\Sigma^{-1}=\lambda I$, the positive risk differences are observed, which is probably due to the error of asymptotic approximation for small $n$. It is found that for $\Sigma^{-1}=\lambda I$ and $\lambda \operatorname{diag}(1,2, \cdots, p), \hat{\Sigma}^{(1)}$ is better than $\hat{\Sigma}_{H}^{(1)}$; for $\Sigma^{-1}=\lambda \operatorname{diag}(1,10$, $\left.\cdots, 10^{p-1}\right), \hat{\Sigma}^{(1)}$ is slightly worse than $\hat{\Sigma}_{H}^{(1)}$; for $\Sigma^{-1}=\lambda \operatorname{diag}(1,0, \cdots, 0)$, the asymptotic differences are consistent up to $O\left(n^{-3}\right)$. The last statement can be confirmed by putting $\Sigma^{-1}=\lambda \operatorname{diag}(1,0, \cdots, 0)$ in Theorems 4.1 and 4.2. From Tables 1, 2 and 5, we can compute the rates of the reduction of the risks of $\hat{\Sigma}^{(1)}$ with respect to

\begin{tabular}{|c|c|c|c|c|c|c|c|}
\hline \multicolumn{2}{|c|}{$\Sigma^{-1}$} & & $n=8$ & $n=16$ & $n=32$ & $n=64$ & $n=128$ \\
\hline \multirow[t]{4}{*}{$p=2$} & $\lambda(1,1)$ & $\begin{array}{l}O\left(n^{-2}\right) \\
O\left(n^{-3}\right) \\
\text { approx. }\end{array}$ & $\begin{array}{c}-.031250 \\
.033854 \\
.003\end{array}$ & $\begin{array}{c}-.007813 \\
-.004232 \\
-.0036\end{array}$ & $\begin{array}{r}-.001953 \\
-.000529 \\
-.00142\end{array}$ & $\begin{array}{r}-.000488 \\
.000066 \\
-.000422\end{array}$ & $\begin{array}{r}-.000122 \\
.000008 \\
-.000114\end{array}$ \\
\hline & $\lambda(1,2)$ & $\begin{array}{l}O\left(n^{-2}\right) \\
O\left(n^{-3}\right) \\
\text { approx. }\end{array}$ & $\begin{array}{c}-.018438 \\
-.008778 \\
-.0097\end{array}$ & $\begin{array}{c}-.004609 \\
-.001097 \\
-.0035\end{array}$ & $\begin{array}{r}-.001152 \\
.000137 \\
-.00102\end{array}$ & $\begin{array}{r}-.000288 \\
-.000017 \\
-.000271\end{array}$ & $\begin{array}{r}-.000072 \\
.000002 \\
-.000070\end{array}$ \\
\hline & $\lambda(1,10)$ & $\begin{array}{l}O\left(n^{-2}\right) \\
O\left(n^{-3}\right) \\
\text { approx. }\end{array}$ & $\begin{array}{c}.005040 \\
-.001753 \\
.0033\end{array}$ & $\begin{array}{c}.001260 \\
-.000219 \\
.00104\end{array}$ & $\begin{array}{r}.000315 \\
-.000027 \\
.000288\end{array}$ & $\begin{array}{r}.000079 \\
-.000003 \\
.000075\end{array}$ & $\begin{array}{r}.000020 \\
-.000000 \\
.000019\end{array}$ \\
\hline & $\lambda(1,0)$ & $\begin{array}{l}O\left(n^{-2}\right) \\
O\left(n^{-3}\right) \\
\text { approx. }\end{array}$ & $\begin{array}{c}.007813 \\
-.000651 \\
.00716\end{array}$ & $\begin{array}{r}.001953 \\
-.000081 \\
.001872\end{array}$ & $\begin{array}{r}.000488 \\
-.000010 \\
.000478\end{array}$ & $\begin{array}{r}.000122 \\
-.000001 \\
.000121\end{array}$ & $\begin{array}{r}.000031 \\
-.000000 \\
.000030\end{array}$ \\
\hline
\end{tabular}
$\hat{\Sigma}_{o}^{(1)}$, namely, $100 \times\left\{R_{1}\left(\hat{\Sigma}_{O}^{(1)}, \Sigma\right)-R_{1}\left(\hat{\Sigma}^{(1)}, \Sigma\right)\right\} / R_{1}\left(\hat{\Sigma}_{O}^{(1)}, \Sigma\right)$ which range above to $20 \%$ for $n \geqq 16$. This may be compared with $8 \%$ for $\hat{\Sigma}_{H}^{(1)}$. If we compare the rates of $\hat{\Sigma}^{(1)}$

Table 5. Asymptotic values of $R_{1}\left(\hat{\Sigma}^{(1)}, \Sigma\right)-R_{1}\left(\hat{\Sigma}_{J S}^{(1)}, \Sigma\right)$ 
Table 5. (continued)

\begin{tabular}{|c|c|c|c|c|c|c|}
\hline$\Sigma^{-1}$ & & $n=8$ & $n=16$ & $n=32$ & $n=64$ & $n=128$ \\
\hline$p=3$ & $\begin{array}{l}O\left(n^{-2}\right) \\
O\left(n^{-3}\right) \\
\text { approx. }\end{array}$ & $\begin{array}{l}-.156250 \\
.153646 \\
-.003\end{array}$ & $\begin{array}{c}-.039063 \\
.019206 \\
-.020\end{array}$ & $\begin{array}{c}-.009766 \\
.002401 \\
-.0074\end{array}$ & $\begin{array}{r}-.002441 \\
.000300 \\
-.00214\end{array}$ & $\begin{array}{r}-.000610 \\
-.000038 \\
-.000573\end{array}$ \\
\hline$\lambda(1,2,3)$ & $\begin{array}{l}O\left(n^{-2}\right) \\
O\left(n^{-3}\right) \\
\text { approx. }\end{array}$ & $\begin{array}{c}-.103316 \\
.069561 \\
-.034\end{array}$ & $\begin{array}{c}-.025829 \\
-.008695 \\
-.0171\end{array}$ & $\begin{array}{c}-.006457 \\
-.001087 \\
-.0054\end{array}$ & $\begin{array}{c}-.001614 \\
.000136 \\
-.00148\end{array}$ & $\begin{array}{r}-.000404 \\
-.000017 \\
-.000387\end{array}$ \\
\hline$\lambda\left(1,10,10^{2}\right)$ & $\begin{array}{l}O\left(n^{-2}\right) \\
O\left(n^{-3}\right) \\
\text { approx. }\end{array}$ & $\begin{array}{c}.021771 \\
-.009179 \\
.0126\end{array}$ & $\begin{array}{c}.005443 \\
-.001147 \\
.0043\end{array}$ & $\begin{array}{c}.001361 \\
-.000143 \\
.00122\end{array}$ & $\begin{array}{r}.000340 \\
-.000018 \\
.000322\end{array}$ & $\begin{array}{r}.000085 \\
-.000002 \\
.000083\end{array}$ \\
\hline$\lambda(1,0,0)$ & $\begin{array}{l}O\left(n^{-2}\right) \\
O\left(n^{-3}\right) \\
\text { approx. }\end{array}$ & $\begin{array}{c}.031250 \\
-.005208 \\
.0260\end{array}$ & $\begin{array}{c}.007813 \\
-.000651 \\
.00716\end{array}$ & $\begin{array}{r}.001953 \\
-.000081 \\
.001872\end{array}$ & $\begin{array}{r}.000488 \\
-.000010 \\
.000478\end{array}$ & $\begin{array}{r}.000122 \\
-.000001 \\
.000121\end{array}$ \\
\hline$\lambda(1, \cdots, 1)$ & $\begin{array}{l}O\left(n^{-2}\right) \\
O\left(n^{-3}\right) \\
\text { approx. }\end{array}$ & $\begin{array}{l}-.406250 \\
. .404297 \\
-.002\end{array}$ & $\begin{array}{c}-.101563 \\
.050537 \\
-.051\end{array}$ & $\begin{array}{c}-.025391 \\
.006317 \\
-.0191\end{array}$ & $\begin{array}{r}-.006348 \\
.000790 \\
-.00556\end{array}$ & $\begin{array}{r}-.001587 \\
.000099 \\
-.001488\end{array}$ \\
\hline$\lambda(1,2,3,4)$ & $\begin{array}{l}O\left(n^{-2}\right) \\
O\left(n^{-3}\right) \\
\text { approx. }\end{array}$ & $\begin{array}{l}-.276042 \\
. .204965 \\
-.07\end{array}$ & $\begin{array}{c}-.069010 \\
.025621 \\
-.043\end{array}$ & $\begin{array}{c}-.017253 \\
.003203 \\
-.0140\end{array}$ & $\begin{array}{r}-.004313 \\
.000400 \\
-.00391\end{array}$ & $\begin{array}{r}-.001078 \\
.000050 \\
-.001208\end{array}$ \\
\hline$\lambda(1$, & $\begin{array}{l}O\left(n^{-2}\right) \\
O\left(n^{-3}\right) \\
\text { approx. }\end{array}$ & $\begin{array}{c}.066391 \\
-.027263 \\
.039\end{array}$ & $\begin{array}{l}.016598 \\
-.003408 \\
.0132\end{array}$ & $\begin{array}{c}.004149 \\
-.000426 \\
.00372\end{array}$ & $\begin{array}{r}.001037 \\
-.000053 \\
.000984\end{array}$ & $\begin{array}{r}.000259 \\
-.000007 \\
.000253\end{array}$ \\
\hline$\lambda(1$, & $\begin{array}{l}O\left(n^{-2}\right) \\
O\left(n^{-3}\right) \\
\text { approx. }\end{array}$ & $\begin{array}{c}.085938 \\
-.017578 \\
.068\end{array}$ & $\begin{array}{c}.021484 \\
-.002197 \\
.0193\end{array}$ & $\begin{array}{c}.005371 \\
-.000275 \\
.00510\end{array}$ & $\begin{array}{r}.00 \\
-.00 \\
.00\end{array}$ & $\begin{array}{r}.000336 \\
-.000004 \\
.000331\end{array}$ \\
\hline$\lambda(1, \cdots, 1)$ & $\begin{array}{l}O\left(n^{-2}\right) \\
O\left(n^{-3}\right) \\
\text { approx. }\end{array}$ & $\begin{array}{c}-.812500 \\
.841667 \\
.03\end{array}$ & $\begin{array}{l}-.203125 \\
.105208 \\
-.10\end{array}$ & $\begin{array}{c}-.050781 \\
.013151 \\
-.038\end{array}$ & $\begin{array}{c}-.012695 \\
.001644 \\
-.0111\end{array}$ & $\begin{array}{c}-.003174 \\
-.000205 \\
-.00297\end{array}$ \\
\hline$\lambda(1,2 \cdots, 5)$ & $\begin{array}{l}O\left(n^{-2}\right) \\
O\left(n^{-3}\right) \\
\text { approx. }\end{array}$ & $\begin{array}{r}-.556302 \\
.435419 \\
-.12\end{array}$ & $\begin{array}{c}-.139075 \\
.054427 \\
-.085\end{array}$ & $\begin{array}{c}-.034769 \\
.006803 \\
-.0280\end{array}$ & $\begin{array}{r}-.008692 \\
.000850 \\
-.00784\end{array}$ & $\begin{array}{r}-.002173 \\
.000106 \\
-.00207\end{array}$ \\
\hline$\lambda\left(1,10, \cdots, 10^{4}\right)$ & $\begin{array}{l}O\left(n^{-2}\right) \\
O\left(n^{-3}\right) \\
\text { approx. }\end{array}$ & $\begin{array}{l}.154470 \\
-.061226 \\
.093\end{array}$ & $\begin{array}{c}.038618 \\
-.007653 \\
.0310\end{array}$ & $\begin{array}{c}.009654 \\
-.000957 \\
.00870\end{array}$ & $\begin{array}{c}.002414 \\
-.000120 \\
.00229\end{array}$ & $\begin{array}{r}.000603 \\
-.000015 \\
.000588\end{array}$ \\
\hline$\lambda(1,0, \cdots, 0)$ & $\begin{array}{l}O\left(n^{-2}\right) \\
O\left(n^{-3}\right) \\
\text { approx. }\end{array}$ & $\begin{array}{c}.187500 \\
-.041667 \\
.146\end{array}$ & $\begin{array}{c}.046875 \\
-.005208 \\
.0417\end{array}$ & $\begin{array}{c}.011719 \\
-.000651 \\
.01107\end{array}$ & $\begin{array}{r}.002930 \\
-.000081 \\
.002848\end{array}$ & $\begin{array}{r}.000732 \\
-.000010 \\
.000722\end{array}$ \\
\hline \multirow[t]{2}{*}{$p=6$} & $\begin{array}{l}O\left(n^{-2}\right) \\
O\left(n^{-3}\right) \\
\text { approx. }\end{array}$ & $\begin{array}{c}-1.406250 \\
1.529948 \\
.1\end{array}$ & $\begin{array}{c}-.351563 \\
.191243 \\
-.16\end{array}$ & $\begin{array}{c}-.087891 \\
.023905 \\
-.064\end{array}$ & $\begin{array}{c}-.021973 \\
.002988 \\
-.0190\end{array}$ & $\begin{array}{r}-.005493 \\
-.000374 \\
-.00512\end{array}$ \\
\hline & $\begin{array}{l}O\left(n^{-2}\right) \\
O\left(n^{-3}\right) \\
\text { approx. }\end{array}$ & $\begin{array}{r}-.963619 \\
.782396 \\
-.18\end{array}$ & $\begin{array}{c}-.240905 \\
.097799 \\
-.143\end{array}$ & $\begin{array}{c}-.060226 \\
.012225 \\
-.048\end{array}$ & $\begin{array}{c}-.015057 \\
.001528 \\
-.0135\end{array}$ & $\begin{array}{r}-.003764 \\
.000191 \\
-.00357\end{array}$ \\
\hline$\lambda\left(1,10, \cdots, 10^{5}\right)$ & $\begin{array}{l}O\left(n^{-2}\right) \\
O\left(n^{-3}\right) \\
\text { approx. }\end{array}$ & $\begin{array}{l}.301591 \\
-.116287 \\
.19\end{array}$ & $\begin{array}{l}.075398 \\
-.014536 \\
.061\end{array}$ & $\begin{array}{c}.018849 \\
-.001817 \\
.0170\end{array}$ & $\begin{array}{c}.004712 \\
-.000227 \\
.00449\end{array}$ & $\begin{array}{r}.001178 \\
-.000028 \\
.001150\end{array}$ \\
\hline$\lambda(1,0, \cdots, 0)$ & $\begin{array}{l}O\left(n^{-2}\right) \\
O\left(n^{-3}\right) \\
\text { approx. }\end{array}$ & $\begin{array}{l}.351563 \\
-.081380 \\
.270\end{array}$ & $\begin{array}{c}.087891 \\
-.010173 \\
.078\end{array}$ & $\begin{array}{c}.021973 \\
-.001272 \\
.0207\end{array}$ & $\begin{array}{c}.005493 \\
-.000159 \\
.00533\end{array}$ & $\begin{array}{r}.001373 \\
-.000020 \\
.001353\end{array}$ \\
\hline
\end{tabular}

with respect to $\hat{\Sigma}_{J S}^{(1)}$, we get the range from $-5.6 \%$ to $12 \%$ in Table 5 for $n \geqq 16$. The rates for $\hat{\Sigma}^{(1)}$ with respect to $\hat{\Sigma}_{H}^{(1)}$ range from $-0.4 \%$ to $12 \%$ for $n \geqq 16$. 


\section{Risks under $L_{2}$ loss}

5.1. Risk of Haff's estimator. We shall now consider the estimator

$$
\hat{\Sigma}_{H}^{(2)}=\frac{1}{n+p+1}\left[S+\frac{b}{\operatorname{tr} S^{-1}} I\right]
$$

proposed by Haff [5], where $C$ is taken to be $I$ in (1.4) without loss of generality. The loss function is given by (1.2), throughout Section 5 . It is known by Haff [5] that the best scalar multiple of $S$ is given by $\hat{\Sigma}_{o}^{(2)}=S /(n+p+1)$. The difference of risks can be written by

$$
\begin{aligned}
& R_{2}\left(\hat{\Sigma}_{H}^{(2)}, \Sigma\right)-R_{2}\left(\hat{\Sigma}_{O}^{(2)}, \Sigma\right) \\
& \quad=\frac{b}{2(n+p+1)^{2}} E\left[\frac{2}{\operatorname{tr} S^{-1}} \operatorname{tr}\left\{S \Sigma^{-2}-(n+p+1) \Sigma^{-1}\right\}+\frac{b \operatorname{tr} \Sigma^{-2}}{\left(\operatorname{tr} S^{-1}\right)^{2}}\right] .
\end{aligned}
$$

To evaluate each expectation, we need the following equations due to Haff [5] derived from the Wishart identity.

$$
\begin{gathered}
E\left[\frac{\operatorname{tr} S \Sigma^{-2}}{\operatorname{tr} S^{-1}}\right]=n E\left[\frac{\operatorname{tr} \Sigma^{-1}}{\operatorname{tr} S^{-1}}\right]+2 E\left[\frac{\operatorname{tr} S^{-1} \Sigma^{-1}}{\left(\operatorname{tr} S^{-1}\right)^{2}}\right] \\
E\left[\frac{\operatorname{tr} S^{-1} \Sigma^{-1}}{\left(\operatorname{tr} S^{-1}\right)^{2}}\right]=(n-p-2) E\left[\frac{\operatorname{tr} S^{-2}}{\left(\operatorname{tr} S^{-1}\right)^{2}}\right]+4 E\left[\frac{\operatorname{tr} S^{-3}}{\left(\operatorname{tr} S^{-1}\right)^{3}}\right]-1 \\
E\left[\frac{\operatorname{tr} \Sigma^{-2}}{\left(\operatorname{tr} S^{-1}\right)^{2}}\right]=4 E\left[\frac{\operatorname{tr} S^{-2} \Sigma^{-1}}{\left(\operatorname{tr} S^{-1}\right)^{3}}\right]+(n-p-1) E\left[\frac{\operatorname{tr} S^{-1} \Sigma^{-1}}{\left(\operatorname{tr} S^{-1}\right)^{2}}\right] .
\end{gathered}
$$

Together with (4.4) and Lemma 4.1, we can rewrite (5.2) as

$$
\begin{aligned}
\frac{b}{(n+p+1)^{2}} & {\left[-n(p+1)+\left\{2 n-4 p-4-b n(p+1)+\frac{b n^{2}}{2}\right\} \frac{\operatorname{tr} \Sigma^{-2}}{\left(\operatorname{tr} \Sigma^{-1}\right)^{2}}+(p+1)^{2}\right.} \\
& \left.-8 \frac{\operatorname{tr} \Sigma^{-3}}{\left(\operatorname{tr} \Sigma^{-1}\right)^{3}}+3(b n+4) \frac{\left(\operatorname{tr} \Sigma^{-2}\right)^{2}}{\left(\operatorname{tr} \Sigma^{-1}\right)^{4}}\right]+O\left(n^{-4}\right) .
\end{aligned}
$$

Assuming that $b=O(1 / n)$, the term of $O\left(n^{-2}\right)$ in (5.6) is

$$
-n(p+1)+2 n\left(1+\frac{b n}{4}\right) \frac{\operatorname{tr} \Sigma^{-2}}{\left(\operatorname{tr} \Sigma^{-1}\right)^{2}} \leqq-n(p+1)+2 n\left(1+\frac{b n}{4}\right) .
$$

The condition that the R.H.S. of (5.7) is negative is given by $b \leqq 2(p-1) / n$ which is in contrast with the exact result $b \leqq 2(p-1) /(n-p+3)$ in Haff [5]. The equality in (5.7) is attained by $\Sigma^{-1}=\lambda \operatorname{diag}(1,0, \cdots, 0)$, for which the value of $(5.6)$ is minimized by

$$
b=\frac{(n-p+1)(p-1)}{n^{2}-2(p-2) n}=\frac{1}{n}(p-1)\left(1+\frac{p-3}{n}\right)+O\left(n^{-3}\right)
$$


Again the result is the same as the optimal choice $b=(p-1) /(n-p+3)$ by Haff [5] asymptotically. Note that

$$
\begin{aligned}
& R_{2}\left(\hat{\Sigma}_{J S}^{(2)}, \Sigma\right)-R_{2}\left(\hat{\Sigma}_{O}^{(2)}, \Sigma\right) \\
& \quad=-\frac{p\left(p^{2}-1\right)}{6 n^{2}}+\frac{p(p+1)^{2}(p-1)}{2 n^{3}}+O\left(n^{-4}\right) .
\end{aligned}
$$

We get

THEOREM 5.1. An asymptotic expansion of the difference of risks between Haff's estimator $\hat{\Sigma}_{H}^{(2)}$ defined by (5.1) and James and Stein's minimax estimator $\hat{\Sigma}_{J}^{(2)}$ for $L_{2}$ loss is given by

$$
\begin{gathered}
R_{2}\left(\hat{\Sigma}_{H}^{(2)}, \Sigma\right)-R_{2}\left(\hat{\Sigma}_{J S}^{(2)}, \Sigma\right)=\frac{p-1}{6 n^{2}}\left[(p+1)(p-6)+3(p+3) \frac{\operatorname{tr} \Sigma^{-2}}{\left(\operatorname{tr} \Sigma^{-1}\right)^{2}}\right] \\
+\frac{p-1}{n^{3}}\left[\frac{1}{2}(p+1)^{2}(6-p)-\Delta(p+1)+3(p+3) \frac{\left(\operatorname{tr} \Sigma^{-2}\right)^{2}}{\left(\operatorname{tr} \Sigma^{-1}\right)^{4}}\right. \\
\left.+(p+1)(\Delta-2 p-6) \frac{\operatorname{tr} \Sigma^{-2}}{\left(\operatorname{tr} \Sigma^{-1}\right)^{2}}-8 \frac{\operatorname{tr} \Sigma^{-3}}{\left(\operatorname{tr} \Sigma^{-1}\right)^{3}}\right]+O\left(n^{-4}\right)
\end{gathered}
$$

where $b=(p-1)(1+\Delta / n) / n$ and an optimal choice of $\Delta$ is $p-3$.

The term of $O\left(n^{-2}\right)$ in $(5.10)$ is the same as that of $R_{1}\left(\hat{\Sigma}_{H}^{(1)}, \Sigma\right)-R_{1}\left(\hat{\Sigma}_{J S}^{(1)}, \Sigma\right)$ in Theorem 4.1. However the term of $O\left(n^{-3}\right)$ is different which yields poor asymptotic approximations as can be seen in Table 6 compared with Table 4 . For instance, when $n=16, p=6$ and $\Sigma^{-1}=\lambda I$, the approximate value of $R_{2}\left(\hat{\Sigma}_{H}^{(2)}, \Sigma\right)-$ $R_{2}\left(\hat{\Sigma}_{J S}^{(2)}, \Sigma\right)$ is equal to -0.032 . However we can not say that this is negative, because of the error that may arise in the asymptotic approximations. The corresponding value for $\hat{\Sigma}_{H}^{(1)}$ is 0.0205 from Table 4 and we are certain that this is positive. One might think that an asymptotic expansion with respect to $n+p+1$ is better for $\hat{\Sigma}_{H}^{(2)}$, because of (3.7), We can easily rewrite (5.10) in terms of powers of $n+p+1$ instead of $n$. For the above example we get the term of order $(n+p+1)^{-2}$ is equal to 0.007089 and the term of order $(n+p+1)^{-3}$ is equal to -0.011290 . The approximate value is -0.004201 , which is different from -0.032 . However still the second term is larger than the first in absolute value. If we increase $n=128$ in this example, the approximate value is 0.000150 , the corresponding value in Table 6 is 0.000138 . Hence these values are reliable. The fact that the asymptotic approximations are better for $L_{1}$ loss than for $L_{2}$ loss, is ascertained again. From Tables 3 and 6 , the rates of the reduction of the risks of $\hat{\Sigma}_{H}^{(2)}$ with respect to $\hat{\Sigma}_{o}^{(2)}$ can be computed, the range of which is given by $0 \% \sim 4 \%$ for $n \geqq 32$ in Table 6. 
Table 6. Asymptotic values of $R_{2}\left(\hat{\Sigma}_{H I}^{(2)}, \Sigma\right)-R_{2}\left(\hat{\Sigma}_{J S}^{(2)}, \Sigma\right)$

\begin{tabular}{|c|c|c|c|c|c|c|}
\hline$\Sigma^{-1}$ & & $n=8$ & $n=16$ & $n=32$ & $n=64$ & $n=128$ \\
\hline$p=2$ & $\begin{array}{l}O\left(n^{-2}\right) \\
O\left(n^{-3}\right) \\
\text { approx. }\end{array}$ & $\begin{array}{c}-.011719 \\
.012207 \\
.0005\end{array}$ & $\begin{array}{c}-.002930 \\
-.001526 \\
-.0014\end{array}$ & $\begin{array}{c}-.000732 \\
-.000191 \\
-.00054\end{array}$ & $\begin{array}{r}-.000183 \\
-.000024 \\
-.000159\end{array}$ & $\begin{array}{r}-.000046 \\
.000003 \\
-.000043\end{array}$ \\
\hline$\lambda(1,2)$ & $\begin{array}{l}O\left(n^{-2}\right) \\
O\left(n^{-3}\right) \\
\text { approx. }\end{array}$ & $\begin{array}{c}-.009549 \\
-.009042 \\
-.0005\end{array}$ & $\begin{array}{c}-.002387 \\
.001130 \\
-.0013\end{array}$ & $\begin{array}{r}-.000597 \\
.000141 \\
-.00046\end{array}$ & $\begin{array}{r}-.000149 \\
-.000018 \\
-.000132\end{array}$ & $\begin{array}{r}-.000037 \\
.000002 \\
-.000035\end{array}$ \\
\hline$\lambda(1,10)$ & $\begin{array}{l}O\left(n^{-2}\right) \\
O\left(n^{-3}\right) \\
\text { approx. }\end{array}$ & $\begin{array}{l}.001356 \\
-.004123 \\
-.0028\end{array}$ & $\begin{array}{r}.000339 \\
-.000515 \\
-.00018\end{array}$ & $\begin{array}{r}.000085 \\
-.000064 \\
.000020\end{array}$ & $\begin{array}{r}.000021 \\
-.000008 \\
.000013\end{array}$ & $\begin{array}{r}.000005 \\
-.000001 \\
.000004\end{array}$ \\
\hline$\lambda(1,0)$ & $\begin{array}{l}O\left(n^{-2}\right) \\
O\left(n^{-3}\right) \\
\text { approx. }\end{array}$ & $\begin{array}{r}.007813 \\
-.009766 \\
-.0020\end{array}$ & $\begin{array}{c}.001953 \\
-.001221 \\
.0007\end{array}$ & $\begin{array}{c}.000488 \\
-.000153 \\
.00034\end{array}$ & $\begin{array}{r}.000122 \\
-.000019 \\
.000103\end{array}$ & $\begin{array}{r}.000031 \\
-.000002 \\
.000028\end{array}$ \\
\hline$\lambda(1,1,1)$ & $\begin{array}{l}O\left(n^{-2}\right) \\
O\left(n^{-3}\right) \\
\text { approx. }\end{array}$ & $\begin{array}{c}-.031250 \\
.035590 \\
.004\end{array}$ & $\begin{array}{c}-.007813 \\
-.004449 \\
-.0034\end{array}$ & $\begin{array}{r}-.001953 \\
.000556 \\
-.00134\end{array}$ & $\begin{array}{r}-.000488 \\
-.000070 \\
-.000419\end{array}$ & $\begin{array}{r}-.000122 \\
.000009 \\
-.000113\end{array}$ \\
\hline$\lambda(1,2,3)$ & $\begin{array}{l}O\left(n^{-2}\right) \\
O\left(n^{-3}\right) \\
\text { approx. }\end{array}$ & $\begin{array}{c}-.026042 \\
.026259 \\
.0002\end{array}$ & $\begin{array}{c}-.006510 \\
-.003282 \\
-.0032\end{array}$ & $\begin{array}{r}-.001628 \\
-.000410 \\
-.00122\end{array}$ & $\begin{array}{r}-.000407 \\
-.000051 \\
-.000356\end{array}$ & $\begin{array}{r}-.000102 \\
.000006 \\
-.000095\end{array}$ \\
\hline$\lambda\left(1,10,10^{2}\right)$ & $\begin{array}{l}O\left(n^{-2}\right) \\
O\left(n^{-3}\right) \\
\text { approx. }\end{array}$ & $\begin{aligned} .014358 \\
-.035581 \\
-.021\end{aligned}$ & $\begin{array}{r}.003590 \\
-.004448 \\
-.0009\end{array}$ & $\begin{array}{c}.000897 \\
-.000556 \\
.00034\end{array}$ & $\begin{array}{r}.000224 \\
-.000069 \\
.000155\end{array}$ & $\begin{array}{r}.000056 \\
-.000009 \\
.000047\end{array}$ \\
\hline$\lambda(1,0,0)$ & $\begin{array}{l}O\left(n^{-2}\right) \\
O\left(n^{-3}\right) \\
\text { approx. }\end{array}$ & $\begin{array}{l}.031250 \\
-.054688 \\
-.023\end{array}$ & $\begin{array}{c}.007813 \\
-.006836 \\
.0010\end{array}$ & $\begin{array}{c}.001953 \\
-.000854 \\
.00110\end{array}$ & $\begin{array}{c}.000488 \\
-.000107 \\
.00038\end{array}$ & $\begin{array}{r}.000122 \\
-.000013 \\
.000109\end{array}$ \\
\hline$\lambda(1, \cdots, 1)$ & $\begin{array}{l}O\left(n^{-2}\right) \\
O\left(n^{-3}\right) \\
\text { approx. }\end{array}$ & $\begin{array}{c}-.037109 \\
-.026733 \\
-.010\end{array}$ & $\begin{array}{c}-.009277 \\
.003342 \\
-.0059\end{array}$ & $\begin{array}{r}-.002319 \\
.000418 \\
-.00190\end{array}$ & $\begin{array}{r}-.000580 \\
-.000052 \\
-.000528\end{array}$ & $\begin{array}{r}-.000145 \\
-.000007 \\
-.000138\end{array}$ \\
\hline$\lambda(1,2,3,4)$ & $\begin{array}{l}O\left(n^{-2}\right) \\
O\left(n^{-3}\right) \\
\text { approx. }\end{array}$ & $\begin{array}{c}-.028906 \\
-.009316 \\
-.0196\end{array}$ & $\begin{array}{c}-.007227 \\
-.001165 \\
-.0061\end{array}$ & $\begin{array}{c}-.001807 \\
-.000146 \\
-.00166\end{array}$ & $\begin{array}{r}-.000452 \\
-.000018 \\
-.000433\end{array}$ & $\begin{array}{r}-.000113 \\
.000002 \\
-.000111\end{array}$ \\
\hline$\lambda\left(1,10,10^{2}, 10^{3}\right)$ & $\begin{array}{l}O\left(n^{-2}\right) \\
O\left(n^{-3}\right) \\
\text { approx. }\end{array}$ & $\begin{array}{l}.056135 \\
-.146300 \\
-.09\end{array}$ & $\begin{array}{l}.014034 \\
-.018288 \\
-.004\end{array}$ & $\begin{array}{c}.003508 \\
-.002286 \\
.0012\end{array}$ & $\begin{array}{c}.000877 \\
-.000286 \\
.00059\end{array}$ & $\begin{array}{r}.000219 \\
-.000036 \\
.000184\end{array}$ \\
\hline$\lambda(1,0,0,0)$ & $\begin{array}{l}O\left(n^{-2}\right) \\
O\left(n^{-3}\right) \\
\text { approx. }\end{array}$ & $\begin{aligned} & .085938 \\
&-.187500 \\
&-.10\end{aligned}$ & $\begin{aligned} .021484 \\
-.023438 \\
-.002\end{aligned}$ & $\begin{array}{c}.005371 \\
-.002930 \\
.0024\end{array}$ & $\begin{array}{r}.001343 \\
-.000366 \\
.00098\end{array}$ & $\begin{array}{r}.000336 \\
-.000046 \\
.000290 \\
\end{array}$ \\
\hline$\lambda(1, \cdots, 1)$ & $\begin{array}{l}O\left(n^{-2}\right) \\
O\left(n^{-3}\right) \\
\text { approx. }\end{array}$ & $\begin{array}{l}-.012500 \\
-.079375 \\
-.092\end{array}$ & $\begin{array}{l}-.003125 \\
-.009922 \\
-.0130\end{array}$ & $\begin{array}{l}-.000781 \\
-.001240 \\
-.0020\end{array}$ & $\begin{array}{l}-.000195 \\
-.000155 \\
-.00035\end{array}$ & $\begin{array}{r}-.000049 \\
-.000019 \\
-.000068\end{array}$ \\
\hline$\lambda(1,2, \cdots, 5)$ & $\begin{array}{l}O\left(n^{-2}\right) \\
O\left(n^{-3}\right) \\
\text { approx. }\end{array}$ & $\begin{array}{l}-.001389 \\
-.106505 \\
-.11\end{array}$ & $\begin{array}{l}-.000347 \\
-.013313 \\
-.014\end{array}$ & $\begin{array}{l}-.000087 \\
-.001664 \\
-.0018\end{array}$ & $\begin{array}{l}-.000022 \\
-.000208 \\
-.00023\end{array}$ & $\begin{array}{r}-.000005 \\
-.000026 \\
-.000031\end{array}$ \\
\hline$\lambda\left(1,10, \cdots, 10^{4}\right)$ & $\begin{array}{l}O\left(n^{-2}\right) \\
O\left(n^{-3}\right) \\
\text { approx. }\end{array}$ & $\begin{array}{r}.142050 \\
-.410155 \\
-.27\end{array}$ & $\begin{aligned} .035512 \\
-.051269 \\
-.016\end{aligned}$ & $\begin{array}{c}.008878 \\
-.006409 \\
.0025\end{array}$ & $\begin{array}{c}.002220 \\
-.000801 \\
.00142\end{array}$ & $\begin{array}{r}.000555 \\
-.000100 \\
.00046\end{array}$ \\
\hline$\lambda(1,0, \cdots, 0)$ & $\begin{array}{l}O\left(n^{-2}\right) \\
O\left(n^{-3}\right) \\
\text { approx. }\end{array}$ & $\begin{aligned} & . 187500 \\
&-.484375 \\
&-.30\end{aligned}$ & $\begin{array}{r}.046875 \\
-.060547 \\
-.014\end{array}$ & $\begin{array}{c}.011719 \\
-.007568 \\
.0042\end{array}$ & $\begin{array}{c}.002930 \\
-.000946 \\
.00198\end{array}$ & $\begin{array}{r}.000732 \\
-.000118 \\
.00061\end{array}$ \\
\hline
\end{tabular}


Table 6. (continued)

\begin{tabular}{|c|c|c|c|c|c|c|}
\hline$\Sigma^{-1}$ & & $n=8$ & $n=16$ & $n=32$ & $n=64$ & $n=128$ \\
\hline$\lambda(1, \cdots, 1)$ & $\begin{array}{l}O\left(n^{-2}\right) \\
O\left(n^{-3}\right) \\
\text { approx. }\end{array}$ & $\begin{aligned} .058594 \\
-.370822 \\
-.31\end{aligned}$ & $\begin{array}{r}.014648 \\
-.046353 \\
-.032\end{array}$ & $\begin{array}{r}.003662 \\
-.005794 \\
-.0021\end{array}$ & $\begin{array}{c}.000916 \\
-.000724 \\
.00019\end{array}$ & $\begin{array}{r}.000229 \\
-.000091 \\
.000138\end{array}$ \\
\hline$\lambda(1,2,$. & $\begin{array}{l}O\left(n^{-2}\right) \\
O\left(n^{-3}\right) \\
\text { approx. }\end{array}$ & $\begin{aligned} .072545 \\
-.409160 \\
-.38\end{aligned}$ & $\begin{array}{l}.018136 \\
-.051145 \\
-.033\end{array}$ & $\begin{array}{r}.004534 \\
-.006393 \\
-.0019\end{array}$ & $\begin{array}{c}.001134 \\
-.000799 \\
.00033\end{array}$ & $\begin{array}{r}.000283 \\
-.000100 \\
.00018\end{array}$ \\
\hline$\lambda(1,10, \cdots, 1$ & $\begin{array}{l}O\left(n^{-2}\right) \\
O\left(n^{-3}\right) \\
\text { approx. }\end{array}$ & $\begin{aligned} & .287643 \\
&-.924538 \\
&-.64\end{aligned}$ & $\begin{aligned} & .071911 \\
&-.115567 \\
&-.04\end{aligned}$ & $\begin{array}{l}.017978 \\
-.014446 \\
.004\end{array}$ & $\begin{array}{c}.004494 \\
-.001806 \\
.0027\end{array}$ & $\begin{array}{c}.001124 \\
-.000226 \\
.00090\end{array}$ \\
\hline$\lambda(1,0, \cdots, 0)$ & $\begin{array}{l}O\left(n^{-2}\right) \\
O\left(n^{-3}\right) \\
\text { approx. }\end{array}$ & $\begin{aligned} & .351563 \\
&-1.044922 \\
&-.7\end{aligned}$ & $\begin{aligned} & .087891 \\
&-.130615 \\
&-.04\end{aligned}$ & $\begin{array}{c}.021973 \\
-.016327 \\
.006\end{array}$ & $\begin{array}{c}.005493 \\
-.002041 \\
.0035\end{array}$ & $\begin{array}{c}.001373 \\
-.000255 \\
.00112\end{array}$ \\
\hline
\end{tabular}

5.2. Risk of new estimator. Finally we shall consider the estimator (1.7) for $C=I$ without loss of generality, namely,

$$
\hat{\Sigma}^{(2)}=\frac{1}{n+p+1}\left(S+\frac{b \operatorname{tr} S^{-1}}{\operatorname{tr} S^{-2}} I\right) .
$$

The risk difference can be written by

$$
R_{2}\left(\hat{\Sigma}^{(2)}, \Sigma\right)-R_{2}\left(\hat{\Sigma}_{O}^{(2)}, \Sigma\right)=\frac{b}{(n+p+1)^{2}} E\left[\frac{\operatorname{tr} S^{-1}}{\operatorname{tr} S^{-2}} \operatorname{tr}\left\{S \Sigma^{-1}-(n+p+1) I\right\} \Sigma^{-1}\right.
$$

$$
\left.+\frac{b}{2}\left(\frac{\operatorname{tr} S^{-1}}{\operatorname{tr} S^{-2}}\right)^{2} \operatorname{tr} \Sigma^{-2}\right]
$$

Each expectation can be computed by the following relations obtained from the Wishart identity in Haff [5],

$$
\begin{gathered}
E\left[\frac{\operatorname{tr} S^{-1} \operatorname{tr} S^{-2} \Sigma^{-1}}{\left(\operatorname{tr} S^{-2}\right)^{2}}\right]=2 E\left[4 \frac{\operatorname{tr} S^{-1} \operatorname{tr} S^{-5}}{\left(\operatorname{tr} S^{-2}\right)^{3}}-\frac{\operatorname{tr} S^{-4}}{\left(\operatorname{tr} S^{-2}\right)^{2}}\right] \\
-2 E\left[\frac{\left(\operatorname{tr} S^{-1}\right)^{2}}{\operatorname{tr} S^{-2}}\right]+(n-p-3) E\left[\frac{\operatorname{tr} S^{-1} \operatorname{tr} S^{-3}}{\left(\operatorname{tr} S^{-2}\right)^{2}}\right] . \\
E\left[\frac{\operatorname{tr} S^{-1} \Sigma^{-1}}{\operatorname{tr} S^{-2}}\right]=n-p-2-E\left[\frac{\left(\operatorname{tr} S^{-1}\right)^{2}}{\operatorname{tr} S^{-2}}\right]+4 E\left[\frac{\operatorname{tr} S^{-4}}{\left(\operatorname{tr} S^{-2}\right)^{2}}\right] . \\
E\left[\frac{\left(\operatorname{tr} S^{-1}\right)^{2}}{\left(\operatorname{tr} S^{-2}\right)^{2}} \operatorname{tr} S^{-1} \Sigma^{-1}\right]=(n-p-2) E\left[\frac{\left(\operatorname{tr} S^{-1}\right)^{2}}{\operatorname{tr} S^{-2}}\right]-E\left[\frac{\left(\operatorname{tr} S^{-1}\right)^{4}}{\left(\operatorname{tr} S^{-2}\right)^{2}}\right] \\
+8 E\left[\frac{\left(\operatorname{tr} S^{-1}\right)^{2} \operatorname{tr} S^{-4}}{\left(\operatorname{tr} S^{-2}\right)^{3}}\right]-4 E\left[\frac{\operatorname{tr} S^{-1} \operatorname{tr} S^{-3}}{\left(\operatorname{tr} S^{-2}\right)^{2}}\right] .
\end{gathered}
$$

For example, the first term of the expectation in the R.H.S. of (5.12) can be expressed by the Whisart identity as 


$$
\begin{aligned}
& n E\left[\frac{\operatorname{tr} S^{-1}}{\operatorname{tr} S^{-2}} \operatorname{tr} \Sigma^{-1}\right]-(n-p-1)(n+p+1) E\left[\frac{\left(\operatorname{tr} S^{-1}\right)^{2}}{\operatorname{tr} S^{-2}}\right]+4 E\left[\frac{\operatorname{tr} S^{-1} \operatorname{tr} S^{-2} \Sigma^{-1}}{\left(\operatorname{tr} S^{-2}\right)^{2}}\right] \\
& -2 E\left[\frac{\operatorname{tr} S^{-1} \Sigma^{-1}}{\operatorname{tr} S^{-2}}\right]-4(n+p+1) E\left[\frac{\operatorname{tr} S^{-1} \operatorname{tr} S^{-3}}{\left(\operatorname{tr} S^{-2}\right)^{2}}\right]+2(n+p+1),
\end{aligned}
$$

which can be reduced further by (5.13), (5.14) and (4.16). Assuming that $b=$ $O\left(n^{-1}\right)$, we can finally rewrite (5.12) as

$$
\begin{gathered}
\frac{b}{(n+p+1)^{2}}\left[-2 n+n\left(\frac{b}{2} n-p-1\right) E\left[\frac{\left(\operatorname{tr} S^{-1}\right)^{2}}{\operatorname{tr} S^{-2}}\right]+4 n E\left[\frac{\operatorname{tr} S^{-1} \operatorname{tr} S^{-3}}{\left(\operatorname{tr} S^{-2}\right)^{2}}\right]\right. \\
+4 p+6+\left\{(p+1)^{2}-6-\frac{b}{2} n(2 p+3)\right\} \frac{\left(\operatorname{tr} \Sigma^{-1}\right)^{2}}{\operatorname{tr} \Sigma^{-2}}-16 \frac{\operatorname{tr} \Sigma^{-4}}{\left(\operatorname{tr} \Sigma^{-2}\right)^{2}} \\
-4(b n+2 p+4) \frac{\operatorname{tr} \Sigma^{-1} \operatorname{tr} \Sigma^{-3}}{\left(\operatorname{tr} \Sigma^{-2}\right)^{2}}+32 \frac{\operatorname{tr} \Sigma^{-1} \operatorname{tr} \Sigma^{-5}}{\left(\operatorname{tr} \Sigma^{-2}\right)^{3}} \\
\left.+8 b n \frac{\left(\operatorname{tr} \Sigma^{-1}\right)^{2} \operatorname{tr} \Sigma^{-4}}{\left(\operatorname{tr} \Sigma^{-2}\right)^{3}}-\frac{b n}{2} \frac{\left(\operatorname{tr} \Sigma^{-1}\right)^{4}}{\left(\operatorname{tr} \Sigma^{-2}\right)^{2}}\right]+O\left(n^{-4}\right) .
\end{gathered}
$$

By (4.18) the term of $O\left(n^{-2}\right)$ in (5.16) is bounded from above by

$$
\left\{\frac{1}{2} b n^{2}-n(p+1)+2 n\right\} \frac{\left(\operatorname{tr} \Sigma^{-1}\right)^{2}}{\operatorname{tr} \Sigma^{-2}}
$$

which is negative only if $b \leqq 2(p-1) / n$. The upper bound (5.17) is attained for $\Sigma^{-1}=\lambda \operatorname{diag}(1,0, \cdots, 0)$ or any permutation of the diagonal elements of it. For this $\Sigma^{-1}$, the risk difference (5.16) can be written by

$$
\frac{b}{(n+p+1)^{2}}\left\{\frac{1}{2} b n^{2}-n(p-1)+(p-1)^{2}-(p-2) b n\right\}+O\left(n^{-4}\right),
$$

which is minimized by $b=(p-1)(1+\Delta / n) / n$ for $\Delta=p-3$ asymptotically. This optimal choice of $b$ is the same as for $\hat{\Sigma}_{H}^{(2)}$. Using (5.9), we get

THEOREM 5.2. An asymptotic expansion of the difference of risks between estimator $\hat{\Sigma}^{(2)}$ defined by (5.11) with $b=(p-1)(1+\Delta / n) / n$ and James and Stein's estimator $\hat{\Sigma}_{J S}^{(2)}$ for $L_{2}$ loss is given by

$$
\begin{aligned}
& R_{2}\left(\hat{\Sigma}^{(2)}, \Sigma\right)-R_{2}\left(\hat{\Sigma}_{J S}^{(2)}, \Sigma\right) \\
& =\frac{p-1}{6 n^{2}}\left[(p-3)(p+4)-3(p+3) \frac{\left(\operatorname{tr} \Sigma^{-1}\right)^{2}}{\operatorname{tr} \Sigma^{-2}}+24 \frac{\operatorname{tr} \Sigma^{-1} \operatorname{tr} \Sigma^{-3}}{\left(\operatorname{tr} \Sigma^{-2}\right)^{2}}\right] \\
& \quad+\frac{p-1}{n^{3}}\left[-\frac{1}{2}(p+1)\left(p^{2}+p-14\right)-2 \Delta+\left(p^{2}+6 p+13-2 \Delta\right) \frac{\left(\operatorname{tr} \Sigma^{-1}\right)^{2}}{\operatorname{tr} \Sigma^{-2}}\right. \\
& \quad+4(\Delta-4 p-1) \frac{\operatorname{tr} \Sigma^{-1} \operatorname{tr} \Sigma^{-3}}{\left(\operatorname{tr} \Sigma^{-2}\right)^{2}}+8 \frac{\operatorname{tr} \Sigma^{-4}}{\left(\operatorname{tr} \Sigma^{-2}\right)^{2}}+2 \frac{\left(\operatorname{tr} \Sigma^{-1}\right)^{4}}{\left(\operatorname{tr} \Sigma^{-2}\right)^{2}}
\end{aligned}
$$




$$
\begin{aligned}
& -\frac{4}{\left(\operatorname{tr} \nu^{\prime-2}\right)^{3}}\left\{-(p-5)\left(\operatorname{tr} \Sigma^{\prime-1}\right)^{2} \operatorname{tr} \nu^{\prime-4}+16 \operatorname{tr} \nu^{\prime-1} \operatorname{tr} \nu^{\prime-5}\right. \\
& \left.+2\left(\operatorname{tr} 2^{\prime-1}\right)^{3} \operatorname{tr} 2^{\prime-3}+8\left(\operatorname{tr} 2^{\prime-3}\right)^{2}\right\} \\
& \left.+96 \frac{\operatorname{tr} \Sigma^{\prime-1} \operatorname{tr} \nu^{-3} \operatorname{tr} \Sigma^{\prime-4}}{\left(\operatorname{tr} \Sigma^{-2}\right)^{4}}\right]+O\left(n^{-4}\right) \text {. }
\end{aligned}
$$

An optimal choice of $\Delta$ is given by $p-3$.

Note that the term of $O\left(n^{-2}\right)$ for $\hat{\Sigma}^{(2)}$ in (5.19) is the same as the corresponding term of Theorem 4.2 for $\hat{\Sigma}^{(1)}$. Also the term of $O\left(n^{-2}\right)$ for $\hat{\Sigma}_{H}^{(2)}$ in Theorem 5.1 is the same as that of Theorem 4.1 for $\hat{\Sigma}_{H}^{(1)}$. Hence the ranges of $O\left(n^{-2}\right)$ in (4.13) and (4.26) hold also for $\hat{\Sigma}_{H}^{(2)}$ and $\hat{\Sigma}^{(2)}$. Asymptotically, the range for $\hat{\Sigma}^{(2)}$ is wider below than that for $\hat{\Sigma}_{H}^{(2)}$. Some numerical values of the risk differences for $\hat{\Sigma}^{(2)}$ are shown in Table 7 . Comparing with Table 6 , we can see that for $\Sigma^{-1}=\lambda I$ and $\lambda \operatorname{diag}(1,2, \cdots, p), \hat{\Sigma}^{(2)}$ is better considerably; for $\Sigma^{-1}=\lambda \operatorname{diag}(1,10, \cdots$, $\left.10^{p-1}\right), \hat{\Sigma}_{H}^{(2)}$ is better and for $\Sigma^{-1}=\lambda \operatorname{diag}(1,0, \cdots, 0)$, they are the same. The last statement can be checked by putting $\Sigma^{-1}=\lambda \operatorname{diag}(1,0, \cdots, 0)$ in (5.10) and (5.19). Comparing with Table 5, we can see that the asymptotic approximations are poor

\begin{tabular}{|c|c|c|c|c|c|c|c|}
\hline \multicolumn{2}{|r|}{$\Sigma^{-1}$} & & $n=8$ & $n=16$ & $n=32$ & $n=64$ & $n=128$ \\
\hline \multirow[t]{4}{*}{$p=2$} & $\lambda(1,1)$ & $\begin{array}{l}O\left(n^{-2}\right) \\
O\left(n^{-3}\right) \\
\text { approx. }\end{array}$ & $\begin{array}{c}-.031250 \\
.039063 \\
.008\end{array}$ & $\begin{array}{c}-.007813 \\
.004883 \\
-.0029\end{array}$ & $\begin{array}{r}-.001953 \\
-.000610 \\
-.00134\end{array}$ & $\begin{array}{r}-.000488 \\
-.000076 \\
-.000412\end{array}$ & $\begin{array}{r}-.000122 \\
.000010 \\
-.000113\end{array}$ \\
\hline & $\lambda(1,2)$ & $\begin{array}{l}O\left(n^{-2}\right) \\
O\left(n^{-3}\right) \\
\text { approx. }\end{array}$ & $\begin{array}{c}-.018438 \\
-.014372 \\
-.004\end{array}$ & $\begin{array}{c}-.004609 \\
-.001796 \\
-.0028\end{array}$ & $\begin{array}{r}-.001152 \\
.000225 \\
-.00093\end{array}$ & $\begin{array}{r}-.000288 \\
-.000028 \\
-.000260\end{array}$ & $\begin{array}{r}-.000072 \\
.000004 \\
-.000069\end{array}$ \\
\hline & $\lambda(1,10)$ & $\begin{array}{l}O\left(n^{-2}\right) \\
O\left(n^{-3}\right) \\
\text { approx. }\end{array}$ & $\begin{array}{r}.005040 \\
-.007077 \\
-.0020\end{array}$ & $\begin{array}{r}.001260 \\
-.000885 \\
.00038\end{array}$ & $\begin{array}{r}.000315 \\
-.000111 \\
.00020\end{array}$ & $\begin{array}{r}.000079 \\
-.000014 \\
.000065\end{array}$ & $\begin{array}{r}.000020 \\
-.000002 \\
.000018\end{array}$ \\
\hline & $\lambda(1,0)$ & $\begin{array}{l}O\left(n^{-2}\right) \\
O\left(n^{-3}\right) \\
\text { approx. }\end{array}$ & $\begin{array}{r}.007813 \\
-.009766 \\
-.0020\end{array}$ & $\begin{array}{c}.001953 \\
-.001221 \\
.0007\end{array}$ & $\begin{array}{c}.000488 \\
-.000153 \\
.00034\end{array}$ & $\begin{array}{r}.000122 \\
-.000019 \\
.000103\end{array}$ & $\begin{array}{r}.000031 \\
-.000002 \\
.000028\end{array}$ \\
\hline \multirow[t]{4}{*}{$p=3$} & $\lambda(1,1,1)$ & $\begin{array}{l}O\left(n^{-2}\right) \\
O\left(n^{-3}\right) \\
\text { approx. }\end{array}$ & $\begin{array}{c}-.156250 \\
.236979 \\
.08\end{array}$ & $\begin{array}{c}-.039063 \\
-.029622 \\
-.009\end{array}$ & $\begin{array}{c}-.009766 \\
-.003703 \\
-.0061\end{array}$ & $\begin{array}{r}-.002441 \\
-.000463 \\
-.00198\end{array}$ & $\begin{array}{r}-.000610 \\
.000058 \\
-.000552\end{array}$ \\
\hline & $\lambda(1,2,3)$ & $\begin{array}{l}O\left(n^{-2}\right) \\
O\left(n^{-3}\right) \\
\text { approx. }\end{array}$ & $\begin{array}{c}-.103316 \\
.128827 \\
.26\end{array}$ & $\begin{array}{c}-.025829 \\
.016103 \\
-.010\end{array}$ & $\begin{array}{c}-.006457 \\
-.002013 \\
-.0044\end{array}$ & $\begin{array}{r}-.001614 \\
-.000252 \\
-.00136\end{array}$ & $\begin{array}{r}-.000404 \\
.000031 \\
-.000372\end{array}$ \\
\hline & $\lambda\left(1,10,10^{2}\right)$ & $\begin{array}{l}O\left(n^{-2}\right) \\
O\left(n^{-3}\right) \\
\text { approx. }\end{array}$ & $\begin{array}{l}.021771 \\
-.042109 \\
-.020\end{array}$ & $\begin{array}{c}.005443 \\
-.005264 \\
.0002\end{array}$ & $\begin{array}{c}.001361 \\
-.000658 \\
.00070\end{array}$ & $\begin{array}{r}.000340 \\
-.000082 \\
.000258\end{array}$ & $\begin{array}{r}.000085 \\
-.000010 \\
.000075\end{array}$ \\
\hline & $\lambda(1,0,0)$ & $\begin{array}{l}O\left(n^{-2}\right) \\
O\left(n^{-3}\right) \\
\text { approx. }\end{array}$ & $\begin{aligned} & .031250 \\
&-.054688 \\
&-.023\end{aligned}$ & $\begin{array}{c}.007813 \\
-.006836 \\
.0010\end{array}$ & $\begin{array}{c}.001953 \\
-.000854 \\
.00110\end{array}$ & $\begin{array}{r}.000488 \\
-.000107 \\
.00038\end{array}$ & $\begin{array}{r}.000122 \\
-.000013 \\
.000109\end{array}$ \\
\hline
\end{tabular}
for $\hat{\Sigma}^{(2)}$. Again the positive values for $\Sigma^{-1}=\lambda I$ and negative values for $\Sigma^{-1}=$

Table 7. Asymptotic values of $R_{2}\left(\hat{\Sigma}^{(2)}, \Sigma^{\prime}\right)-R_{2}\left(\hat{\Sigma}_{J S}^{(2)}, \Sigma^{\prime}\right)$ 
Table 7. (continued)

\begin{tabular}{|c|c|c|c|c|c|c|}
\hline$\Sigma^{-1}$ & & $n=8$ & $n=16$ & $n=32$ & $n=64$ & $n=128$ \\
\hline$\lambda(1, \cdots, 1)$ & $\begin{array}{l}O\left(n^{-2}\right) \\
O\left(n^{-3}\right) \\
\text { approx. }\end{array}$ & $\begin{array}{c}-.406250 \\
.708984 \\
.30\end{array}$ & $\begin{array}{c}-.101563 \\
.088623 \\
-.013\end{array}$ & $\begin{array}{c}-.025391 \\
-.011078 \\
-.014\end{array}$ & $\begin{array}{c}-.006348 \\
.001385 \\
-.0050\end{array}$ & $\begin{array}{r}-.001587 \\
-.000173 \\
-.00141\end{array}$ \\
\hline$\lambda(1,2,3,4)$ & $\begin{array}{l}O\left(n^{-2}\right) \\
O\left(n^{-3}\right) \\
\text { approx. }\end{array}$ & $\begin{array}{l}-.276042 \\
.419957 \\
.14\end{array}$ & $\begin{array}{c}-.069010 \\
.052495 \\
-.017\end{array}$ & $\begin{array}{c}-.017253 \\
.006562 \\
-.0107\end{array}$ & $\begin{array}{c}-.004313 \\
.000820 \\
-.00349\end{array}$ & $\begin{array}{r}-.001078 \\
-.000103 \\
-.00098\end{array}$ \\
\hline$\lambda\left(1,10,10^{2}, 10^{3}\right)$ & $\begin{array}{l}O\left(n^{-2}\right) \\
O\left(n^{-3}\right) \\
\text { approx. }\end{array}$ & $\begin{array}{r}.066391 \\
-.155830 \\
-.09\end{array}$ & $\begin{array}{r}.016598 \\
-.019479 \\
-.003\end{array}$ & $\begin{array}{c}.004149 \\
-.002435 \\
.0017\end{array}$ & $\begin{array}{c}.001037 \\
-.000304 \\
.00073\end{array}$ & $\begin{array}{r}.000259 \\
-.000038 \\
.000221\end{array}$ \\
\hline$\lambda(1,0,0,0)$ & $\begin{array}{l}O\left(n^{-2}\right) \\
O\left(n^{-3}\right) \\
\text { approx. }\end{array}$ & $\begin{array}{r}.085938 \\
-.187500 \\
-.10\end{array}$ & $\begin{array}{l}.021484 \\
-.023438 \\
-.002\end{array}$ & $\begin{array}{l}.005371 \\
-.002930 \\
.0024\end{array}$ & $\begin{array}{c}.001343 \\
-.000366 \\
.00098\end{array}$ & $\begin{array}{r}.000336 \\
-.000046 \\
.000290\end{array}$ \\
\hline$\lambda(1, \cdots, 1)$ & $\begin{array}{l}O\left(n^{-2}\right) \\
O\left(n^{-3}\right) \\
\text { approx. }\end{array}$ & $\begin{array}{c}-.812500 \\
1.590625 \\
.8\end{array}$ & $\begin{array}{c}-.203125 \\
.198828 \\
-.004\end{array}$ & $\begin{array}{c}-.050781 \\
.024854 \\
-.026\end{array}$ & $\begin{array}{c}-.012695 \\
.003107 \\
-.0096\end{array}$ & $\begin{array}{r}-.003174 \\
.000388 \\
-.00279\end{array}$ \\
\hline$\lambda(1,2, \cdots, 5)$ & $\begin{array}{l}O\left(n^{-2}\right) \\
O\left(n^{-3}\right) \\
\text { approx. }\end{array}$ & $\begin{array}{c}-.556302 \\
.968478 \\
.41\end{array}$ & $\begin{array}{l}-.139075 \\
. .121060 \\
-.02\end{array}$ & $\begin{array}{c}-.034769 \\
-.015132 \\
-.020\end{array}$ & $\begin{array}{c}-.008692 \\
-.001892 \\
-.0068\end{array}$ & $\begin{array}{r}-.002173 \\
.000236 \\
-.00194\end{array}$ \\
\hline$\lambda\left(1,10, \cdots, 10^{4}\right)$ & $\begin{array}{l}O\left(n^{-2}\right) \\
O\left(n^{-3}\right) \\
\text { approx. }\end{array}$ & $\begin{array}{l}.154470 \\
-.421797 \\
-.27\end{array}$ & $\begin{array}{l}.038618 \\
-.052725 \\
-.014\end{array}$ & $\begin{array}{c}.009654 \\
-.006591 \\
.0031\end{array}$ & $\begin{array}{c}.002414 \\
-.000824 \\
.00159\end{array}$ & $\begin{array}{r}.000603 \\
-.000103 \\
.00050\end{array}$ \\
\hline$\lambda(1,0, \cdots, 0)$ & $\begin{array}{l}O\left(n^{-2}\right) \\
O\left(n^{-3}\right) \\
\text { approx. }\end{array}$ & $\begin{array}{r}.187500 \\
-.484375 \\
-.30\end{array}$ & $\begin{array}{l}.046875 \\
-.060547 \\
-.014\end{array}$ & $\begin{array}{c}.011719 \\
-.007568 \\
.0042\end{array}$ & $\begin{array}{c}.002930 \\
-.000946 \\
.00198\end{array}$ & $\begin{aligned} .000732 \\
-.000118 \\
.00061\end{aligned}$ \\
\hline$\lambda(1, \cdots, 1)$ & $\begin{array}{l}O\left(n^{-2}\right) \\
O\left(n^{-3}\right) \\
\text { approx. }\end{array}$ & $\begin{array}{l}-1.406250 \\
3.040365 \\
1.6\end{array}$ & $\begin{array}{c}-.351563 \\
.380046 \\
.03\end{array}$ & $\begin{array}{l}-.087891 \\
.047506 \\
-.040\end{array}$ & $\begin{array}{c}-.021973 \\
.005938 \\
-.0160\end{array}$ & $\begin{array}{r}-.005493 \\
.000742 \\
-.00475\end{array}$ \\
\hline$\lambda(1,2, \cdots, 6)$ & $\begin{array}{l}O\left(n^{-2}\right) \\
O\left(n^{-3}\right) \\
\text { approx. }\end{array}$ & $\begin{array}{c}-.963619 \\
1.865664 \\
.9\end{array}$ & $\begin{array}{l}-.240905 \\
-.233208 \\
-.01\end{array}$ & $\begin{array}{l}-.060226 \\
-.029151 \\
-.031\end{array}$ & $\begin{array}{c}-.015057 \\
-.003644 \\
-.0114\end{array}$ & $\begin{array}{r}-.003764 \\
-.000455 \\
-.00331\end{array}$ \\
\hline$\lambda\left(1,10, \cdots, 10^{5}\right)$ & $\begin{array}{l}O\left(n^{-2}\right) \\
O\left(n^{-3}\right) \\
\text { approx. }\end{array}$ & $\begin{aligned} & .301591 \\
&-.936962 \\
&-.64\end{aligned}$ & $\begin{array}{l}.075398 \\
-.117120 \\
-.04\end{array}$ & $\begin{array}{l}.018849 \\
-.014640 \\
.004\end{array}$ & $\begin{array}{c}.004712 \\
-.001830 \\
.0029\end{array}$ & $\begin{array}{r}.001178 \\
-.000229 \\
.00095\end{array}$ \\
\hline$\lambda(1,0, \cdots, 0)$ & $\begin{array}{l}O\left(n^{-2}\right) \\
O\left(n^{-3}\right) \\
\text { approx. }\end{array}$ & $\begin{array}{l}.351563 \\
-1.044922 \\
-.7\end{array}$ & $\begin{array}{l}.087891 \\
-.130615 \\
-.04\end{array}$ & $\begin{array}{l}.021973 \\
-.016327 \\
.003\end{array}$ & $\begin{array}{c}.005493 \\
-.002041 \\
.0035\end{array}$ & $\begin{array}{r}.001373 \\
-.000255 \\
.00112\end{array}$ \\
\hline
\end{tabular}

$\lambda \operatorname{diag}(1,0, \cdots, 0)$ when $n=8$ or 16 in Table 7 are doubtful. From Tables 3 and 7 , we can compute the rates of the reduction of the risks for $\hat{\Sigma}^{(2)}$ with respect to $\hat{\Sigma}_{o}^{(2)}$, which range above to $11 \%$ for $n \geqq 32$. This may be compared with $4 \%$ for $\hat{\Sigma}_{H}^{(2)}$. Comparing the rates for $\hat{\Sigma}^{(2)}$ with respect to $\hat{\Sigma}_{H}^{(2)}$, the range is given by $-0.2 \% \sim 7 \%$ for $n \geqq 32$ in Table 7 . Also the rates for $\hat{\Sigma}^{(2)}$ with respect to $\hat{\Sigma}_{J S}^{(2)}$ range $-1.2 \% \sim 8 \%$ while the rates for $\hat{\Sigma}_{H}^{(2)}$ with respect to $\hat{\Sigma}_{J S}^{(2)}$ range only $-1.2 \%$ $\sim 0.8 \%$ for $n \geqq 32$.

Acknowledgment. The authors wish to express their gratitude to the referee for his careful reading the manuscript and useful comments. 


\section{References}

[1] Abramowitz, M. and Stegun, I. A., Handbook of Mathematical Functions. NBS App. Math. Ser. 551964.

[2] Fujikoshi, Y., Asymptotic expansions of the distributions of test statistics in multivariate analysis. J. Sci. Hiroshima Univ. Ser. A-I 34 (1970) 73-144.

[3] Giri, N., Multivariate Statistical Inference. Academic, 1977.

[4] Haff, L. R., An identity for the Wishart distribution with applications. J. Multivariate Anal. 9 (1979) 531-544.

[5] Haff, L. R., Empirical Bayes estimation of the multivariate normal covariance matrix. Ann. Statist. 8 (1980) 586-597.

[6] Ito, K., Asymptotic formulae for the distribution of Hotelling's generalized $\mathrm{T}_{0}^{2}$ statistic. Ann. Math. Statist. 27 (1956) 1091-1105.

[7] James, W. and Stein, C., Estimation with quadratic loss. Fourth Berkeley Symp. Math. Statist. Probability, Univ. California Press Berkeley 1961.

[ 8 ] Kendall, M.G. and Stuart, A., The Advanced Theory of Statistics. Vol. 2 3rd Edition, Griffin 1973.

[9] Kshirsagar, A. M., Multivariate Analysis. Marcel Dekker 1972.

[10] Nagao, H., On some test criteria for covariance matrix. Ann. Statist. 1 (1973) 700-709.

[11] Okamoto, M., An asymptotic expansion for the distribution of the linear discriminant function. Ann. Math. Statist. 34 (1963) 1286-1301.

[12] Selliah, J., Estimation and testing problems in a Wishart distribution. Ph. D. thesis, Dept. Statist. Stanford Univ. 1964.

[13] Sharma, D., An estimator of normal covariance matrix. Calcutta Statist. Assoc. Bulletin 29 (1980) 161-167.

[14] Siotani, M., On the distribution of the Hotelling's $T^{2}$-statistic. Ann. Inst. Statist. Math. 8 (1957) 1-14.

[15] Sugiura, N., Derivatives of the characteristic root of a symmetric or a hermitian matrix with two applications in multivariate analysis. Commun. Statist. 1 (1973) 393-417.

Institute of Mathematics

University of Tsukuba

Ibaraki 305 Japan 